\title{
Der Mindestlohn im Dachdeckerhandwerk: Auswirkungen auf Beschäftigung, Arbeitnehmerschutz und Wettbewerb
}

\author{
Bodo Aretz • Melanie Arntz • Terry Gregory • \\ Christian Rammer
}

Angenommen: 30. Juli 2012 / Online publiziert: 11. September 2012

(C) Institut für Arbeitsmarkt- und Berufsforschung 2012

Zusammenfassung Der seit 1997 im Dachdeckerhandwerk eingeführte und seit 2003 bundeseinheitlich geregelte, allgemeinverbindliche Mindestlohn führt vor allem in Ostdeutschland auch im internationalen Vergleich zu einer starken Betroffenheit der Branche vom Mindestlohn. Die damit einhergehende effektive Kostenbelastung fällt dennoch begrenzt aus. Auf der Basis von Differenz-von-DifferenzenSchätzungen sowohl im Vergleich zu einer nicht von einem Mindestlohn betroffenen Baunebenbranche als auch auf Basis eines Vergleichs von unterschiedlich stark durch den Mindestlohn betroffenen Beschäftigten des Dachdeckerhandwerks, werden die kausalen Wirkungen im Hinblick auf Beschäftigung, Arbeitnehmerschutz und Wettbewerb untersucht. Dabei zeigt sich, dass sich die mit dem Mindestlohn einhergehenden Lohnzuwächse nur teilweise in Einkommenszuwächse übersetzen. Zudem lassen sich trotz einiger negativer Beschäftigungsergebnisse für die von einem bindenden Mindestlohn betroffenen Beschäftigten keine Veränderung der Gesamtbeschäftigung feststellen. Dies liegt möglicherweise daran, dass mindestlohnbedingte Kos-

Für die in diesem Beitrag publizierten Ergebnisse und Schlussfolgerungen zeichnen ausschließlich die Autorinnen und Autoren verantwortlich. Sie geben nicht notwendigerweise die Position des Bundesministerium für Arbeit und Soziales wieder.

B. Aretz · M. Arntz · T. Gregory $(\bowtie) \cdot$ C. Rammer

ZEW, Mannheim, Deutschland

e-mail: gregory@zew.de

B. Aretz

e-mail: aretz@zew.de

C. Rammer

e-mail: rammer@zew.de

M. Arntz

University of Heidelberg, Heidelberg, Deutschland

e-mail: arntz@zew.de tensteigerungen zumindest teilweise über höhere Preise an die Kunden weitergegeben wurden. Eindeutige Effekte auf die Wettbewerbssituation im Dachdeckerhandwerk konnten hingegen nicht nachgewiesen werden, wenngleich sich für Ostdeutschland eine gewisse Verschiebung der Gründungstätigkeit und des Unternehmensbestands in Richtung EinPersonen-Unternehmen zeigt.

Schlüsselwörter Mindestlohn · Dachdeckerhandwerk · Beschäftigung · Arbeitnehmerschutz · Wettbewerb

JEL Klassifikationen $\mathrm{J} 31 \cdot \mathrm{J} 20 \cdot \mathrm{L} 11$

The minimum wage in the roofing sector: impact on employment, worker protection and competition

Abstract The Minimum wage in the roofing sector, which was introduced in 1997 and transferred to a national and generally binding level in 2003, has particularly affected the sector in Eastern Germany, even in an international comparison. However, the concomitant effective cost burden is limited. Based on difference-in-differences estimations in comparison to an ancillary construction sector that is not affected by minimum wages as well as based on a comparison of workers in the roofing sector who have been affected by minimum wage introduction to different degrees, causal effects with regard to employment, worker protection and competition are investigated. The results show that the wage increases associated with the minimum wage introduction only partially compile into earnings increases. Moreover, the overall employment situation does not seem to change even though some negative employment results can be detected for workers affected by binding minimum wages. This might be due to the fact that, at least partially, cost increases caused 
by minimum wage introduction are transferred to the customers by increasing prices. Distinct effects on the competitive situation cannot be shown; however, there is a certain shift in the East German start-up activity and corporate structure toward single-person companies.

\section{Einleitung}

Der vorliegende Beitrag gibt einen Überblick über die Ergebnisse der Evaluation der Auswirkungen des Mindestlohns im Dachdeckerhandwerk, wo bereits zum 1. Oktober 1997 ein Mindestlohn eingeführt wurde. Seitdem wurde der Mindestlohn mehrfach angehoben und ist zudem seit 2003 bundeseinheitlich geregelt, was zu einer in Ostdeutschland hohen Betroffenheit der Branche vom Mindestlohn geführt hat. Im Jahr 2010 erhielten dort gut $50 \%$ der Beschäftigten den Mindestlohn von 10,80 Euro.

Trotz dieser hohen Betroffenheit sind negative Beschäftigungswirkungen jedoch keine zwangsläufige Folge, sondern hängen mit den Güter- und Arbeitsmarktbedingungen in der Branche zusammen. So können im Falle eines Wettbewerbs um Arbeitskräfte potenziell negative Beschäftigungswirkungen auftreten, während im Fall eines beschränkten Wettbewerbs um Arbeitskräfte auch positive Beschäftigungseffekte denkbar sind (vgl. den Übersichtsartikel von Neumark and Wascher 2008). Ob überhaupt Beschäftigungswirkungen eintreten, hängt jedoch unter anderem von den Möglichkeiten ab, mindestlohnbedingte Lohnkostensteigerungen durch Preiserhöhungen an die Kunden weiterzugeben oder durch Maßnahmen zur Produktivitätssteigerung aufzufangen.

Auf Basis eines Branchenbildes werden zunächst die Wettbewerbsbedingungen auf dem Güter- und Arbeitsmarkt dargestellt und daraus Hypothesen zu möglichen Wirkungen des Mindestlohns abgeleitet. Anschließend werden die kausalen Wirkungen des Mindestlohns im Hinblick auf Beschäftigung, Entlohnungs- und Arbeitsbedingungen sowie auf die Wettbewerbssituation in der Branche analysiert. Hierfür wird ein Kontrollgruppenansatz verwendet, der zwei Kontrollgruppen einschließt: (1) Unternehmen und Beschäftigte einer nicht vom Mindestlohn betroffenen, aber dem Dachdeckerhandwerk möglichst ähnlichen Branche und (2) Unternehmen und Beschäftigte des Dachdeckerhandwerks, die aufgrund einer höheren Entlohnung nicht direkt vom Mindestlohn betroffenen sind.

Für diese Analysen steht mit den administrativen Arbeitnehmer-Arbeitgeberdaten der Bundesagentur für Arbeit, dem Mannheimer Unternehmenspanel des ZEW sowie der Vollerhebung der Beschäftigten des Dachdeckerhandwerks durch die Lohnausgleichskasse des Dachdeckerhandwerks eine sehr gute Datengrundlage zur Verfügung. Insbesondere kann die Betroffenheit vom Mindestlohn wesentlich genauer festgestellt und für kausale Wirkungsanalysen genutzt werden, als dies in der bislang einzigen Evaluationsstudie für Deutschland für das Bauhauptgewerbe von König und Möller (2008) möglich war. ${ }^{1}$

Die Ergebnisse der Evaluation zeigen, dass es vor allem in Ostdeutschland mindestlohnbedingt $\mathrm{zu}$ deutlichen Stundenlohnzuwächsen für die unteren Dezile der Lohnverteilung kam. Dies verschlechterte zwar die Beschäftigungschancen der mindestlohnbedingt verteuerten Arbeitskräfte, ließ die Gesamtbeschäftigung jedoch vermutlich unverändert. Eindeutige Wettbewerbswirkungen ließen sich ebenfalls nicht nachweisen, wenngleich sich für Ostdeutschland eine gewisse Verschiebung der Unternehmensstruktur in Richtung Ein-Personen-Unternehmen zeigt. Auf der Ebene der Preise liegen Hinweise vor, dass die mindestlohnbedingten Kostensteigerungen zumindest teilweise über höhere Preise an die Kunden weitergegeben wurden. In Ostdeutschland wurde ein Teil der mindestlohnbedingten Kostenzuwächse zudem durch eine Lohnzurückhaltung für die oberen Lohngruppen kompensiert.

\section{Der Markt für Dachdeckerleistungen in Deutschland}

Der Gütermarkt für Dachdeckerleistungen setzt sich aus dem Neubaugeschäft, der Sanierung bzw. Reparatur von Dächern und dem Dachausbau einschließlich der Errichtung von Solaranlagen auf Dächern zusammen. Im Gegensatz zu häufig in der Mindestlohn-Literatur untersuchten Dienstleistungsbranchen wie z. B. der Fast-Food-Industrie (Card und Krueger 1994) zeichnet sich das Dachdeckerhandwerk durch einen hohen Anteil an Fachkräften von etwa $65 \%$ aus (Aretz et al. 2011). Der typische Beschäftigte des Dachdeckerhandwerks ist der männliche Vollzeitbeschäftigte mit einer beruflichen Ausbildung. Gleichzeitig machen Personalkosten nur etwa $40 \%$ der Gesamtkosten aus (Kostenstrukturerhebung 2001). Bei hochwertigen Dachsystemen kann der Anteil der Personalkosten aufgrund steigender Materialkosten sogar niedriger ausfallen. Produktivitätsfortschritte können erzielt werden, wenn neue Dachsysteme mit vereinfachten Anbringungs- und Verlegungstechniken auf den Markt gebracht werden.

Das Dachdeckerhandwerk wird vor allem von traditionellen Dachdeckerunternehmen dominiert, die als stehendes Gewerbe dem Meisterzwang unterliegen und meist nicht mehr als 20 Mitarbeiter beschäftigen. Sie bieten ihre Leistungen vielfach regional und hauptsächlich für Privatkunden an. Die Preiselastizität dieses Kundensegments ist vermut-

\footnotetext{
${ }^{1}$ Der Studie von Rattenhuber (2011), die die Wirkungen des Mindestlohns im Bauhauptgewerbe untersucht, stehen zwar mit den Daten der Verdienststrukturerhebung exakte Stundenlohninformationen zur Verfügung; ein Nachteil des Datensatzes ist jedoch zum einen, dass Unternehmen mit mehr als 10 Beschäftigten nicht im Datensatz enthalten sind und zum anderen ist der aktuelle Rand nicht erfasst.
} 


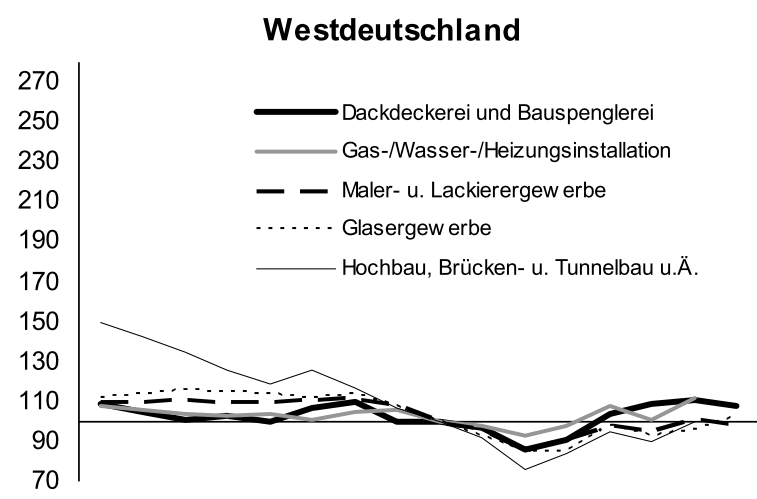

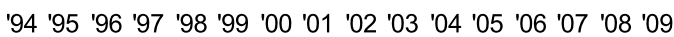

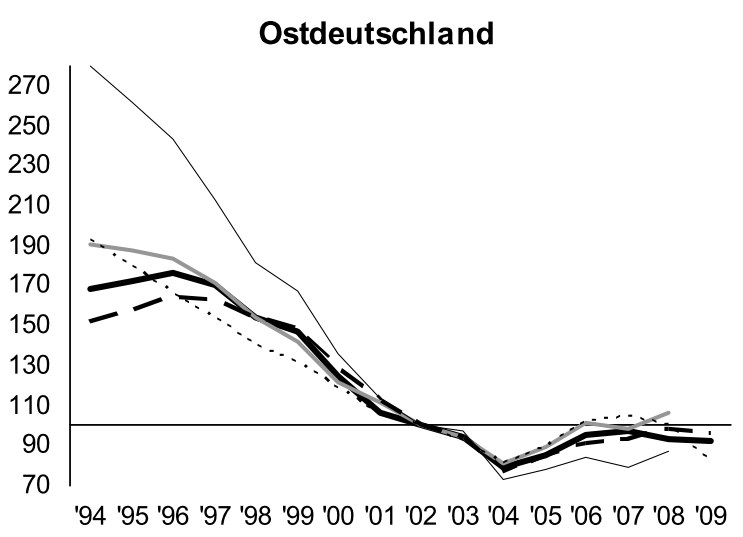

Hochbau, Brücken- u. Tunnelbau u. Ä. wegen mangelnder Vergleichbarkeit der Branchenabgrenzung nach der WZ-Umstellung nur bis 2008

mit zunächst nicht dafür, dass der Wettbewerb um Arbeitskräfte nennenswert eingeschränkt ist.

Andererseits ist zu konstatieren, dass sich das Verhältnis von Arbeitsangebot und Arbeitsnachfrage mit dem Beschäftigungsrückgang in der Dachdeckerbranche zumindest bis vor wenigen Jahren verschlechtert hat. So unterlag das gesamte Baugewerbe nach dem Ende des wiedervereinigungsbedingten Baubooms einem Strukturwandel, der sich insbesondere in Ostdeutschland in rückläufigen Umsätzen (vgl. Abb. 1), starkem Beschäftigungsabbau und einem abnehmenden Verhältnis zwischen Branchenumsatz und Anzahl der Unternehmen niederschlug. Im Unterschied zum Bauhauptgewerbe (Hochbau) erfuhren das Dachdeckerhandwerk und weitere Branchen des Baunebengewerbes weniger starke Umsatzrückgänge vor 2004 sowie eine schnellere wirtschaftliche Erholung nach dem konjunkturellen Tiefpunkt. ${ }^{3}$ Der starke Umsatzeinbruch im Bauhauptgewerbe spiegelt das Einsetzen des langjährigen Abwärtstrends bei den Bauinvestitionen wider, während die schnellere Erholung in den restlichen Branchen auf konjunkturelle Sondereffekte wie dem Aufkommen neuer Geschäftsfelder in den Bereichen Gebäudesanierung und Solartechnik zurückzuführen ist. Während die Umsätze im westdeutschen Dachdeckerhandwerk seit 2005 wieder über dem Niveau von Mitte der 1990er Jahre liegen, hat sich die Entwicklung in Ostdeutschland ab 2004 nur leicht verbessert, die Umsatzwerte aus der Zeit vor der Mindestlohneinführung bleiben weiterhin außer Reichweite.

Die konjunkturelle Entwicklung schlug sich auch auf dem Arbeitsmarkt in Form von hohen Beschäftigungsver-

\footnotetext{
${ }^{3}$ Folgende Wirtschaftszweigklassifikation identifizieren die Branchen: Dachdeckerhandwerk (WZ93/WZ03: 45.22.1), Installationsgewerbe (WZ93: 45.33.1 und 45.33.2./WZ03: 45.33.0), Maler- und Lackiererhandwerk (WZ93/WZ03: 45.44.1), Glasergewerbe (WZ93/WZ03: 45.44.2).
}

${ }^{2}$ Für nähere Informationen zum MUP-Datensatz siehe Anhang A. 

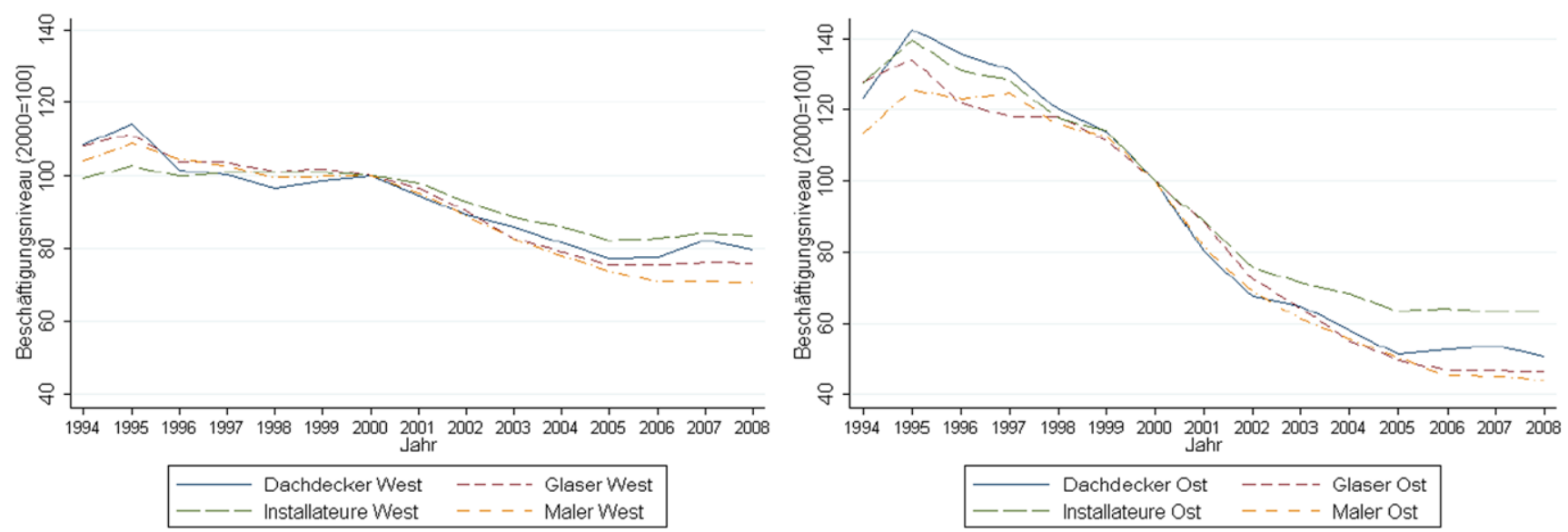

Abb. 2 Entwicklung des Beschäftigungsniveaus nach Branche für West- und Ostdeutschland, 1994-2008 (2000 = 100), BA-Daten (vgl. Anhang A)

lusten, vor allem in Ostdeutschland, nieder, die sich erst seit 2005 stabilisiert haben, vgl. Abb. 2. Der starke Beschäftigungsrückgang kann die Marktposition der Arbeitnehmer schwächen, wenn ehemalige Beschäftigte der Branche aufgrund von Such- und Mobilitätskosten nicht in andere Branchen und/oder Regionen abwandern (vgl. Manning 2003). Ein Wechsel in andere Baubranchen wurde jedoch durch die insgesamt schlechte Marktsituation im Baugewerbe erschwert. In der Unternehmensbefragung gaben gut $60 \%$ der Dachdeckerunternehmer an, dass ihre Beschäftigten im Falle einer Lohnkürzung ihrer Einschätzung nach das Unternehmen verlassen würden. Immerhin $40 \%$ der Unternehmen gehen somit davon aus, schlechtere Arbeitsbedingungen durchsetzen zu können ohne Arbeitnehmer an konkurrierende Unternehmen verlieren zu müssen. Dies kann als ein Indiz dafür gewertet werden, dass der Wettbewerb am Arbeitsmarkt zumindest teilweise beschränkt ist.

Parallel zum Beschäftigungsrückgang nahm die Zahl der Betriebe im gleichen Zeitraum leicht zu. Der Beschäftigungsrückgang ging mit einer Entwicklung hin zu immer kleineren Unternehmensstrukturen einher. So kam es im Dachdeckerhandwerk zu einer starken Zunahme von EinPersonen-Unternehmen (EPU). Nach Auswertungen der Lohnausgleichskasse stieg der Anteil von EPU von etwa $8 \%$ im Jahr 1995 auf $23 \%$ im Jahr 2010. Parallel dazu nahm die Relation zwischen dem Gesamtumsatz der Branche und der Anzahl der Unternehmen und damit die Marktkonzentration ab. Eine zunehmende Zahl an Unternehmen stand somit einem sinkenden Branchenumsatz gegenüber.

\section{Mindestlohnregelungen im Dachdeckerhandwerk}

Der im Oktober 1997 eingeführte allgemeinverbindliche Mindestlohn hat für alle Betriebe und selbstständige Betriebsabteilungen des Dachdeckerhandwerks in Deutsch- land Gültigkeit, die überwiegend Bauleistungen im Sinne des \$211 Absatz 1 SGB III ${ }^{4}$ ausüben. Laut Tarifvertrag ist der persönliche Geltungsbereich des Mindestlohns auf gewerbliche Arbeitnehmer/Innen (Arbeiter/Innen) beschränkt, bei denen es sich weder um Azubis, Reinigungskräfte noch minderjährige Beschäftigte handelt. Kaufmännische Angestellte, die nicht den gewerblichen Beschäftigten zuzurechnen sind, fallen somit ebenfalls nicht in den Geltungsbereich des Mindestlohns. Der Geltungsbereich gilt zudem auch für geringfügig Beschäftigte sowie Leiharbeitnehmer/innen. ${ }^{5}$

Abbildung 3 zeigt die Höhe des allgemeinverbindlichen Mindestlohns im Dachdeckerhandwerk im Zeitablauf. Unmittelbar nach der ersten Allgemeinverbindlichkeitserklärung des Mindestlohns gab es zunächst zwei unterschiedlich hohe Mindestlöhne in Ost- und Westdeutschland. Erst seit 2003 gibt es einen bundeseinheitlichen Mindestlohntarif. Seit seiner Einführung wurde der Mindestlohn mehrfach erhöht, zuletzt am 1. Januar 2012 auf 11 Euro pro Stunde. Seit 2000 wurden die Mindestlohnregelungen für das Dachdeckerhandwerk zudem wiederholt per Rechtsverordnung erneuert. Dabei kam es zwischen den auslaufenden Regelungen und der Erneuerung zu einigen kürzeren Zeitabschnitten ohne Mindestlohnregelung. Mit Ausnahme der einjährigen Unterbrechung 2000/2001 sind diese temporären Unterbrechungen allerdings so kurz, dass kaum mit Anpassungsprozessen im Hinblick auf Beschäftigung, Wettbewerb und Arbeitnehmerschutz zu rechnen ist, zumal Unternehmen jederzeit von einem Fortbestand der Regelung ausgehen konnten

\footnotetext{
${ }^{4}$ „Ein Betrieb des Baugewerbes ist ein Betrieb, der gewerblich überwiegend Bauleistungen auf dem Baumarkt erbringt. Bauleistungen sind alle Leistungen, die der Herstellung, Instandsetzung, Instandhaltung, Änderung oder Beseitigung von Bauwerken dienen. [...]“ (\$211 Absatz 1 SGB III)

${ }^{5}$ Das Arbeitnehmer-Entsendegesetz legt die Tätigkeit und nicht den arbeitsrechtlichen Status als Maßstab für den Geltungsbereich zugrunde (vgl. §8 Abs. 3 AEntG).
} 
Abb. 3 Der

allgemeinverbindliche

Mindestlohn im

Dachdeckerhandwerk

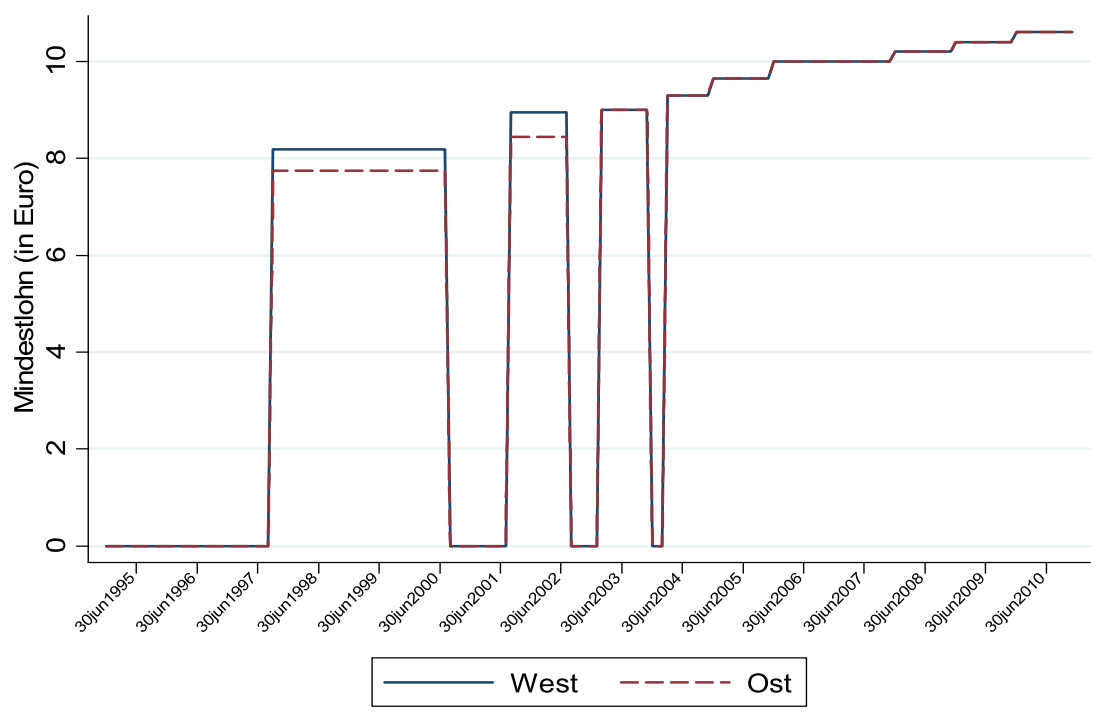

und viele Anpassungsprozesse erst mit Verzögerungen eintreten.

\section{Evaluationsdesign und Datenbasis}

Um die kausalen Wirkungen des Mindestlohns auf die Beschäftigungssituation, den Arbeitnehmerschutz sowie die Wettbewerbssituation im Dachdeckerhandwerk zu identifizieren, gilt es die Frage zu beantworten, wie sich der Mindestlohn auf diese Zielgrößen verglichen mit einer hypothetischen Situation ohne den Mindestlohn ausgewirkt hat. Diese kontrafaktische Situation ohne Mindestlohn wird in der Evaluationsliteratur üblicherweise durch eine Kontrollgruppe gemessen, die der vom Mindestlohn betroffenen so genannten Treatmentgruppe möglichst ähnlich ist. In den USA wurden in der neueren Literatur häufig Unternehmen bzw. Beschäftigte aus einem Bundesstaat mit einem gleichbleibenden Mindestlohnniveau als Kontrollgruppe für Unternehmen bzw. Beschäftigte derselben Branche aus einem Bundesstaat mit einem veränderten Mindestlohnniveau genutzt (vgl. Card 1992; Neumark und Wascher 1992; Card und Krueger 1994; Dube et al. 2010).

In der deutschen Dachdeckerbranche wurde der Mindestlohn jedoch zeitgleich für alle Betriebe des Dachdeckerhandwerks wirksam. Zwar gab es in der Zeit von 1997 bis 2002 in Ost- und Westdeutschland unterschiedliche Mindestlohnhöhen. Ein Vergleich dieser Regionen ist aufgrund des stark abweichenden konjunkturellen Verlaufs (vgl. Abb. 1) jedoch nicht sinnvoll. Somit stehen keine geeigneten Unternehmen bzw. Beschäftigten der Dachdeckerbranche zur Verfügung, die nicht in den Geltungsbereich des Mindestlohns fallen oder von einem anderen Mindestlohnniveau betroffen wären. Für die statistische Rekonstruktion der kontrafaktischen Situation ohne Mindestlohn stehen damit vor allem zwei Kontrollgruppen zur Verfügung:
(1) Unternehmen und Beschäftigte einer dem Dachdeckerhandwerk möglichst ähnlichen, nicht vom Mindestlohn betroffenen Branche und (2) Unternehmen und Beschäftigte des Dachdeckergewerbes selbst, die nicht oder in geringerem Ausmaße von mindestlohnbedingten Lohnveränderungen betroffen sind.

Von allen Baubranchen, die ähnliche konjunkturelle und institutionelle Rahmenbedingungen auf dem Gütermarkt aufweisen und zudem zumindest zum Zeitpunkt der Mindestlohneinführung im Dachdeckerhandwerk von keinem allgemeinverbindlichen Mindestlohn betroffen waren, weist das Installationsgewerbe die größte Ähnlichkeit zum Dachdeckerhandwerk auf und stellt daher die präferierte Kontrollbranche dar (siehe Aretz et al. 2011). Andere potenzielle Vergleichsbranchen wie das Maler- und Lackiererhandwerk (für den Zeitraum vor Einführung eines Mindestlohns in diesem Sektor im Jahr 2003) und das Glasergewerbe schieden u. a. deshalb aus, weil sie anders als das Dachdecker- und Installationshandwerk gar nicht oder deutlich weniger von der energetischen Gebäudesanierung und der Solarförderung profitieren. Für die Unternehmen des Maler- und Lackiererhandwerks kommt hinzu, dass sie aufgrund der Möglichkeit der Eigenarbeit im Bereich des Privatkundengeschäfts unter einem deutlich höheren Preisdruck stehen, sodass sich viele Unternehmen auf Arbeiten für gewerbliche Auftraggeber (Objektkundengeschäft) konzentriert haben und dadurch eine deutlich andere Auftraggeberstruktur aufweisen. Zudem besteht auch im Hinblick auf strukturelle Merkmale (Kostenstruktur, Größenstruktur, Produktivität, Personalkostenanteil), welche den Einfluss des Mindestlohns moderieren können, die größte Übereinstimmung zwischen der Installationsbranche und dem Dachdeckerhandwerk. Diese Ähnlichkeit der Branchen schlägt sich zudem in weitgehend parallelen Trends in Bezug auf Unternehmensanzahl, Umsatz, Preise, Beschäftigung, Gründungen und Schließungen 
vor der Mindestlohneinführung nieder. Für Ansatz (1) wurden daher die Beschäftigten und Unternehmen des Installationsgewerbes zu Vergleichszwecken herangezogen. Details zu der Auswahl der Kontrollbranche können in Aretz et al. (2011) nachgelesen werden.

Durch die Bildung der Differenzen der Zielgrößen der vom Mindestlohn betroffenen Unternehmen oder Beschäftigten vor und nach der Mindestlohneinführung mit den Differenzen der Zielgrößen der jeweiligen Kontrollgruppe (Differenzen-von-Differenzen-Design, kurz: DvD-Design) können zeitkonstante Unterschiede zwischen den beiden Gruppen ebenso herausgefiltert werden wie Einflüsse von Zeittrends, die beide Gruppen in gleichem Maße betreffen. Der kausale Effekt kann somit unter der Annahme identifiziert werden, dass die Veränderungen über die Zeit in beiden Gruppen parallel verlaufen wären, wenn der Mindestlohn nicht eingeführt worden wäre (,,common trend assumption"). Darüber hinaus muss die Kontrollgruppe durch das Treatment unbeeinflusst sein (Stable Unit Treatment Value Assumption (SUTVA), vgl. Cameron und Trivedi 2005: 872). Die Validität dieser Annahme ist jedoch fraglich, wenn so genannte Spillover-Effekte zu Abhängigkeiten zwischen der Treatment- und Kontrollgruppe führen und die Kontrollgruppe somit indirekt ebenfalls von dem Treatment beeinflusst ist.

Für die Analysen greifen wir auf unterschiedliche Datensätze zurück, die in Anhang A kurz beschrieben werden. Für die deskriptiven Analysen zu den Arbeits- und Gütermarktbedingungen wurde neben der amtlichen Statistik (Umsatzsteuerstatistik, Betriebsstatistik, Kostenstrukturerhebung, Statistik der Baukonjunktur und der Baupreise) das Mannheimer Unternehmenspanel (MUP) ausgewertet. Letzteres beinhaltet für jedes Unternehmen einige Strukturmerkmale wie z. B. Wirtschaftszweigkennung, Umsatz (ab 1999), Anzahl der Mitarbeiter und die Bonitätseinstufung durch die Wirtschaftsauskunftei Creditreform. Darüber hinaus wurden zu Beginn der Evaluation eine Reihe qualitativer Interviews mit Unternehmen der Dachdeckerbranche durchgeführt, um Handlungs- und Rahmenbedingungen der Branche zu erfragen und damit eine computergestützte Telefonbefragung (CATI) bei 500 Unternehmen vorzubereiten. Diese Befragung diente der Ergänzung der Sekundärdaten, um Datenlücken im Hinblick auf mögliche Auswirkungen des Mindestlohns zu schließen. Die Arbeitsmarktsituation im Dachdeckerhandwerk wurde zudem auch unter Verwendung administrativer Individualdaten analysiert. Diese bilden neben dem MUP die wichtigste Datengrundlage für die Wirkungsanalysen.

Bei den administrativen Daten handelt es sich um kombinierte Arbeitnehmer-Arbeitgeberdaten. Zum einen steht mit den Daten der Lohnausgleichskasse des Dachdeckerhandwerks (LAK) eine Vollerhebung der Beschäftigten und Betriebe des Dachdeckerhandwerks zur Verfügung. Die wich- tigsten Variablen umfassen den monatlichen Bruttolohn sowie die monatlich entlohnte Arbeitszeit in Stunden, die eine exakte Berechnung des Bruttostundenlohns ermöglichen. Mittels dieser Daten lässt sich die Betroffenheit der Beschäftigten vom Mindestlohn somit wesentlich genauer feststellen, als dies in der bislang einzigen MindestlohnEvaluationsstudie für Deutschland für das Bauhauptgewerbe von König und Möller (2008) möglich war, die auf Daten der Bundesagentur für Arbeit aufbaut. Diese Daten haben insbesondere den Nachteil, dass eine genaue Angabe über die Arbeitsstunden - und somit über die exakten Stundenlöhne - fehlt. Dennoch verwenden wir auch die Daten der Beschäftigtenhistorik und des Betriebshistorikpanels der Bundesagentur für Arbeit (BA), da diese im Gegensatz zu den LAK-Daten auch Beschäftigte und Unternehmen der Installationsbranche beinhaltet. Der oben beschriebene Kontrollgruppenansatz (1) kann somit nur auf Basis dieser Daten umgesetzt werden. Die Verwendung beider Datensätze ermöglicht uns, die Bruttostundenlöhne in den BA-Daten auf Basis eines Schätzmodells zu rekonstruieren. Details zu dem Imputationsverfahren finden sich in Anhang B.

\section{Die Betroffenheit der Branche vom Mindestlohn}

Das durch den Kaitz-Index gemessene Verhältnis vom Mindestlohn zum Medianlohn gibt einen ersten Anhaltspunkt zur Betroffenheit der Branche vom Mindestlohn. Der Index gibt an, in welchem Maße der Mindestlohn im Falle einer Einhaltung der Mindestlohnregelungen in die Lohnverteilung eingreift und zu einer Stauchung der Lohnverteilung führt. Auf der Ebene des einzelnen Beschäftigten lässt sich die Betroffenheit vom Mindestlohn hingegen daran festmachen, ob vor der Mindestlohneinführung bzw. erhöhung eine Entlohnung unterhalb des Mindestlohnniveaus vorlag und somit mindestlohnbedingt ein über das normale Lohnwachstum hinausgehender Lohnanstieg erfolgen müsste. In diesem Fall spricht die Literatur auch von einem bindenden Mindestlohn bzw. einem durch den Mindestlohn betroffenen Beschäftigten (vgl. Card 1992; Card und Krueger 1995). Zum anderen kann das Ausmaß der Betroffenheit für Personen mit einem bindenden Mindestlohn durch die individuelle Lohnlücke, d.h. die prozentuale Differenz zwischen dem tatsächlichen Stundenlohn des Beschäftigten, $w_{i t}$, und dem im Laufe des nächsten Jahres geltenden Mindestlohn $M L_{i, t+1}$, gemessen werden. Um den Grad der (Nicht-)Einhaltung der Mindestlohnregelungen abzuschätzen, wird die Lohnlücke mit der tatsächlichen Lohnveränderung der Beschäftigten im selben Zeitraum verglichen. $\mathrm{Ob}$ die beobachteten Lohnzuwächse der Beschäftigten mit einem bindenden Mindestlohn über das normale Lohnwachstum hinausgehen, lässt sich anhand eines Vergleiches mit den Lohnveränderungen der Beschäftigten ohne einen bin- 
Tab. 1 Individuelle

Betroffenheit von der

Mindestlohneinführung bzw.

nächsten Mindestlohnerhöhung

(1997-2008), LAK-Daten

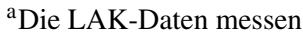
jeweils den Stand im Juni vor der nächsten Mindestlohnstufe

${ }^{\mathrm{b}}$ Die Lohnlücke bezeichnet die durchschnittliche prozentuale Differenz zwischen dem tatsächlichen Stundenlohn des Beschäftigten im Juni und dem im Laufe des nächsten Jahres geltenden Mindestlohn

${ }^{\mathrm{c}}$ Die Lohnveränderung bezeichnet die tatsächliche prozentuale Lohnveränderung zwischen dem Juni vor und dem Juni nach der Einführung der nächsten Mindestlohnstufe

${ }^{\mathrm{d}}$ Der Kaitz-Index gibt das Verhältnis zwischen Mindestlohnhöhe und Medianlohn an

\begin{tabular}{|c|c|c|c|c|c|c|}
\hline \multirow{3}{*}{$\begin{array}{l}\text { Datum der } \\
\text { nächsten } \\
\text { Mindestlohnstufe }\end{array}$} & \multirow{3}{*}{$\begin{array}{l}\text { ML } \\
\text { (in } \\
\text { Euro) }\end{array}$} & \multicolumn{4}{|c|}{ Beschäftigte mit einem bindendem Mindestlohn? } & \multirow{3}{*}{$\begin{array}{l}(5) \\
\text { Kaitz } \\
\text { Index }^{d}\end{array}$} \\
\hline & & \multicolumn{3}{|l|}{$\mathrm{Ja}$} & Nein & \\
\hline & & $\begin{array}{l}\text { (1) } \\
\text { Anteil }^{\mathrm{a}} \\
(\text { in \%) }\end{array}$ & $\begin{array}{l}\text { (2) } \\
\text { Individuelle } \\
\text { Lohnlücke }^{\text {b }} \\
\text { (in \%) }\end{array}$ & $\begin{array}{l}\text { (3) } \\
\text { Lohnveränderung } \\
\text { (in \%) }\end{array}$ & $\begin{array}{l}(4) \\
\text { Lohnveränderung }^{c} \\
(\text { in \%) }\end{array}$ & \\
\hline
\end{tabular}

Westdeutschland

\begin{tabular}{|c|c|c|c|c|c|c|}
\hline 01. Okt 97 & 8,2 & 3,9 & 17,2 & 3,4 & 2,3 & 65,2 \\
\hline 01. Sep 01 & 9,0 & 3,2 & 16,9 & 5,5 & 1,4 & 67,6 \\
\hline 01. Mrz 03 & 9,0 & 3,1 & 15,9 & 5,6 & 2,4 & 67,7 \\
\hline 01. Apr 04 & 9,3 & 4,1 & 14,0 & 4,6 & 1,4 & 68,9 \\
\hline 01. Mai 05 & 9,7 & 4,9 & 14,3 & 4,2 & 0,6 & 70,9 \\
\hline 01. Jan 06 & 10,0 & 5,9 & 15,2 & 3,7 & 1,1 & 73,3 \\
\hline 01. Jan 07 & 10,0 & 8,4 & 11,3 & 5,3 & 3,2 & 73,4 \\
\hline 01. Jan 08 & 10,2 & 9,0 & 9,9 & 4,4 & 2,2 & 74,0 \\
\hline 01. Jan 09 & 10,4 & 8,4 & 9,2 & 9,2 & 3,0 & 74,3 \\
\hline \multicolumn{7}{|c|}{ Ostdeutschland } \\
\hline 01. Okt 97 & 7,7 & 13,1 & 11,0 & 6,6 & 0,0 & 82,2 \\
\hline 01. Sep 01 & 8,4 & 14,4 & 6,6 & 5,5 & 0,7 & 89,7 \\
\hline 01. Mrz 03 & 9,0 & 33,9 & 5,3 & 4,4 & 0,1 & 95,1 \\
\hline 01. Apr 04 & 9,3 & 45,4 & 4,3 & 4,2 & 0,3 & 98,1 \\
\hline 01. Mai 05 & 9,7 & 47,8 & 4,9 & 4,2 & 0,1 & 99,4 \\
\hline 01. Jan 06 & 10,0 & 51,7 & 5,0 & 4,2 & 0,0 & 100,5 \\
\hline 01. Jan 07 & 10,0 & 47,4 & 2,0 & 2,0 & 0,9 & 99,8 \\
\hline 01. Jan 08 & 10,2 & 54,7 & 3,0 & 3,4 & 1,3 & 100,8 \\
\hline 01. Jan 09 & 10,4 & 51,7 & 2,7 & 3,5 & 0,7 & 100,4 \\
\hline
\end{tabular}

denden Mindestlohn abschätzen. Ist dies der Fall, sollte es zu einer Stauchung der Lohnverteilung kommen.

Die Auswertung dieser Indikatoren in Tab. 1 weisen insbesondere in Ostdeutschland auf eine starke Betroffenheit vom Mindestlohn hin. So unterlagen nach der Einführung eines bundeseinheitlichen Mindestlohns in Ostdeutschland zuletzt mehr als die Hälfte der im ostdeutschen Dachdeckerhandwerk gewerblichen abhängigen Beschäftigten einem bindenden Mindestlohn, d.h. sie erhielten in dem Jahr vor der nächsten Mindestlohnerhöhung einen Lohn unterhalb des Mindestlohns (Spalte 1). Das Ausmaß der Eingriffsintensität in Ostdeutschland zeigt sich auch am hohen KaitzWert von zuletzt $100 \%$ und demonstriert, dass der Medianlohn mittlerweile dem Mindestlohn entspricht (Spalte 5). Zum Vergleich variiert der Kaitz-Index für flächendeckende Mindestlöhne in den OECD-Ländern lediglich zwischen Werten von unter 30 Prozent in der Türkei und mehr als 70 Prozent in Italien (Dolton und Rosazza-Bondibene 2011). Auch wenn ein Branchenmindestlohn nicht mit einem flächendeckenden Mindestlohn gleichzusetzen ist, suggerieren diese Zahlen eine auch im internationalen Vergleich hohe Betroffenheit vom Mindestlohn im ostdeutschen Dachdeckerhandwerk. In Westdeutschland waren im Jahr 2009 hingegen maximal $9 \%$ der Beschäftigten betroffen, was sich auch in einem geringeren, wenn auch mit etwa $70 \%$ immer noch hohen Kaitz-Index niederschlägt.

Zudem zeigt sich, dass die Lohnzuwächse der Beschäftigten mit einem bindenden Mindestlohn (Spalte 3) im Vergleich zu Beschäftigten, für die der Mindestlohn nicht bindend war (Spalte 4), vor allem in Ostdeutschland deutlich überdurchschnittlich ausfielen. Dies führte in Ostdeutschland auch im Vergleich zur Installationsbranche $\mathrm{zu}$ einer starken Stauchung der Lohnverteilung (siehe Abb. 4). Für die Lohnverteilung in Westdeutschland lässt sich dagegen eine solche Stauchung nicht erkennen. Allerdings war das Ausmaß der Nicht-Einhaltung der Mindestlohnregelungen in Westdeutschland relativ hoch, denn die Lohnzuwächse der Beschäftigten mit einem bindenden Mindestlohn waren zwar überdurchschnittlich, entsprach jedoch in vielen Jahren nur etwa einem Drittel der durchschnittlichen individuellen Lohnlücke zum Mindestlohnniveau (Spalte 2). Erst seit 2006 nahm die Einhaltung des Mindestlohns in Westdeutschland deutlich und sprunghaft zu, was vermutlich auf verstärkte Kontrollbemühungen in den letzten Jahren zurückzuführen ist. In Ostdeutschland entsprachen die durchschnittlichen Lohnanstiege der von einem bindenden Mindestlohn Betroffenen dagegen weitestgehend der durchschnittlichen individuellen Lohnlücke. 
Abb. 4 Lohnverteilung in der Dachdecker- und Installationsbranche nach Westund Ostdeutschland, 1994 und 2008, BA-Daten
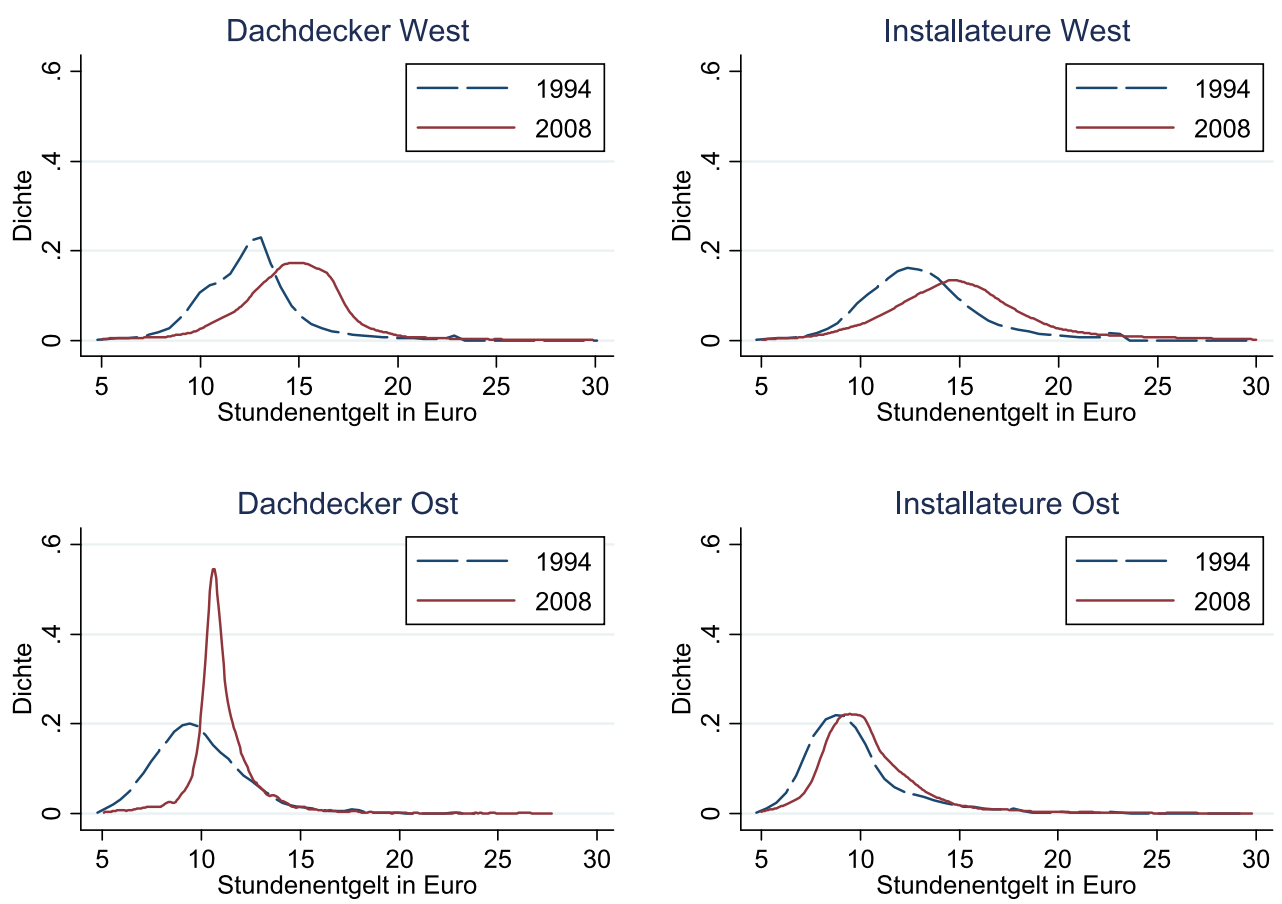

In Westdeutschland waren vom Mindestlohn primär Ungelernte, geringfügig oder in Teilzeit Beschäftigte mit deutlich unterdurchschnittlichen Betriebszugehörigkeiten von einem bindenden Mindestlohn betroffen. In Ostdeutschland hingegen erhält der vom Mindestlohn betroffene Beschäftigte nahezu den durchschnittlichen Stundenlohn. Zwar sind auch in Ostdeutschland ungelernte Arbeitskräfte überdurchschnittlich häufig vom Mindestlohn betroffen, die deutliche Mehrheit der Betroffenen ist jedoch eine vollzeitbeschäftigte Fachkraft.

Trotz der insgesamt hohen Betroffenheit vom Mindestlohn und der insbesondere in Ostdeutschland zu beobachtenden Lohnkompression ist die mit dem Mindestlohn einhergehende effektive Kostenbelastung überschaubar. Zum einen steigen die gesamten Lohnkosten für gewerbliche Beschäftigte selbst bei einer vollständigen Einhaltung des Mindestlohns mit jeder Mindestlohnstufe um lediglich etwa $1 \%$ in Westdeutschland und bis zu 2,5\% in Ostdeutschland. Da die Personalkosten für gewerblich Beschäftigte jedoch nur etwa $30 \%$ der Gesamtkosten ausmachen, relativieren sich die Kosten weiter. So führt der Mindestlohn je nach Mindestlohnstufe selbst bei vollständiger Einhaltung zu Gesamtkostenerhöhungen von lediglich $0,2-0,5 \%$ in West- und $0,3-0,8 \%$ in Ostdeutschland. Zwar relativieren diese Zahlen den Einfluss, den der Mindestlohn im Dachdeckerhandwerk auf Beschäftigung und Wettbewerb auszuüben vermag. Einzelne Betriebe können jedoch durchaus von stärkeren Lohnkostenanstiegen betroffen sein. Zudem kann sich die Kostenbelastung im Laufe der sukzessiven Mindestlohnerhöhungen kumulieren.

\section{Hypothesen zu den Mindestlohnwirkungen im Dachdeckerhandwerk}

Für die von einem bindenden Mindestlohn betroffenen gewerblichen Beschäftigten des Dachdeckerhandwerks ist von Lohnerhöhungen auszugehen, falls die Mindestlohnregelungen wie in Abschn. 3 bereits diskutiert zumindest teilweise eingehalten werden. In Ostdeutschland ist aufgrund der höheren Eingriffsintensität und der stärkeren Einhaltung der Mindestlohnregelungen mit stärkeren Lohnzuwächsen für die betroffenen Beschäftigten zu rechnen als in Westdeutschland. Ob sich entsprechende Lohnzuwächse jedoch in Einkommenszuwächse übersetzen, hängt nicht zuletzt von den Beschäftigungswirkungen des Mindestlohns ab. Denn ein oberhalb des Marktlohnes liegender Mindestlohn stellt zwar diejenigen Arbeitnehmer und Arbeitnehmerinnen besser, die weiterhin beschäftigt bleiben, mögliche Entlassungen als Reaktion könnten aber die durchschnittliche Einkommensposition verschlechtern. Ebenso könnten Arbeitszeitkürzungen das Einkommen - im Gegensatz zum Lohnsatz - der bereits Beschäftigten verringern (vgl. Michl 1996).

Einer mindestlohnbedingten Reduktion der Gesamtbeschäftigung wirkt jedoch entgegen, dass die effektive Kostenbelastung für die Unternehmen trotz der hohen Betroffenheit vom Mindestlohn relativ gering ausfällt. Zudem liegen aufgrund eines beschränkten Preiswettbewerbs auf dem Gütermarkt und eines zumindest partiell beschränkten Wettbewerbs auf dem Arbeitsmarkt Rahmenbedingungen vor, die es eventuell ermöglichen, steigende Arbeitskosten teilweise an die Kunden in Form höherer Preise und an die 
Beschäftigten in Form verschlechterter Arbeitsbedingungen weiterzugeben (Neumark und Wascher 2008). Denkbar ist insbesondere, dass die Arbeitsbelastungen steigen, weil Arbeit verdichtet wird und dadurch (unbezahlte) Überstunden geleistet werden müssen, dass freiwillige Arbeitgeberleistungen zurückgenommen werden oder dass verstärkt weniger geschützte Beschäftigungsformen - befristete Arbeitsverhältnisse, Leiharbeit - zum Einsatz kommen.

Selbst eine unveränderte Gesamtbeschäftigung kann jedoch mit Beschäftigungseffekten für einzelne Beschäftigtengruppen einhergehen. So können veränderte relative Preise zwischen Nichtfachkräften, für die der Mindestlohn häufig bindend ist, und Fachkräften die relative Nachfrage nach Letzteren begünstigen. Aufgrund solcher Substitutionseffekte kann sich zudem auch die Entlohnung von Arbeitskräften verbessern, deren Entlohnung oberhalb des Mindestlohnes liegt. Falls Unternehmen über eine gewisse Marktmacht gegenüber Arbeitnehmern und Arbeitnehmerinnen verfügen, würden Fachkräfte jedoch auch ohne entsprechende Lohnzuwächse nicht zwangsläufig das Unternehmen verlassen. Die zu erwartenden Wirkungen auf die Entlohnung dieser Beschäftigten ist somit keineswegs klar, auch wenn die Literatur vor allem positive Effekte nachweisen konnte (DiNardo et al. 1996; Lee 1999; Teulings 2000).

Mögliche Auswirkungen des Mindestlohns auf den Gütermarkt und die Form und Intensität des Wettbewerbs hängen ebenfalls von den Rahmenbedingungen auf dem Markt für Dachdeckerleistungen ab. Falls die Nachfrage- und Wettbewerbsverhältnisse wie bereits angeklungen eine zumindest partielle Weitergabe der mindestlohnbedingten Kostensteigerung an die Kunden erlauben, so kann der Mindestlohn zu Preiserhöhungen für Dachdeckerleistungen führen. Diese führt wiederum - in Abhängigkeit von der Preiselastizität der Nachfrage - zu einem Rückgang der Nachfrage oder einer Verringerung der Konsumentenrente. In dem Maße, indem zusätzliche Arbeitskosten jedoch nicht überwälzt werden können, können sich die Gewinne der Unternehmen reduzieren, sofern die Unternehmen die mindestlohnbedingte Kostensteigerung nicht durch eine höhere Produktivität kompensieren können. Niedrigere Gewinne verringern die Attraktivität für Marktzutritte und können langfristig zu einer steigenden Zahl von Marktaustritten führen.

Ein Mindestlohn kann aber auch zum Aufbau von Markteintrittsbarrieren für potenzielle Neugründungen führen. In diesem Fall nutzen die etablierten Unternehmen den Mindestlohn als ein Instrument zur Erhöhung der Kosten für potenzielle Wettbewerber (vgl. Göddeke et al. 2011; Haucap et al. 2001). Durch den Mindestlohn wird verhindert, dass wenig produktive Unternehmen über eine Niedrigpreisstrategie in den Wettbewerb eintreten können. Wenn für Neugründungen die Arbeitsproduktivität ihrer Mitarbeiter aufgrund geringerer Erfahrung, geringeren Möglichkeiten zur Nutzung von Skaleneffekten und einer geringeren Kapitalaus- stattung unter dem Mindestlohnniveau liegt, sind Markteintritte unattraktiv und unterbleiben. Markteintrittsbarrieren verringern den Wettbewerbsdruck der etablierten Unternehmen und können so zu einer Verringerung der Marktaustritte und einer Erhöhung der Gewinne führen.

\section{Wirkungen des Mindestlohns}

Die im Rahmen der Wirkungsanalysen betrachteten ZielgröBen betreffen eine Vielzahl an Indikatoren aus den Bereichen Beschäftigung, Arbeitnehmerschutz und Wettbewerbssituation. Im Rahmen dieses Beitrags können jedoch nur zentrale Ergebnisse für ausgewählte Indikatoren dargestellt werden. Für Details verweisen wir auf Aretz et al. (2011).

\subsection{Beschäftigung}

Im Hinblick auf Beschäftigungswirkungen betrachten wir zunächst die Wahrscheinlichkeit, dass eine Beschäftigung in der Branche auch im nächsten Jahr fortgesetzt wird. Aufgrund der konjunkturell schlechten Situation in der Baubranche, die mit einem deutlichen Beschäftigungsrückgang einhergeht, ist diese Weiterbeschäftigungswahrscheinlichkeit eine interessante Größe. Eine mindestlohnbedingte $\mathrm{Zu}$ rückhaltung bei der Einstellung von Beschäftigten wird auf diese Weise jedoch nicht erfasst. Daher wird zusätzlich auf Betriebsebene die Entwicklung des Beschäftigungsumfangs, d.h. Anzahl der gewerblich Beschäftigten, näher beleuchtet. ${ }^{6}$ Die so identifizierten Beschäftigungseffekte bilden sowohl ein verändertes Einstellungs- als auch Entlassungsverhalten der Betriebe ab, können jedoch nur eingeschränkt mit der Entwicklung der Gesamtbeschäftigung in der Branche gleichgesetzt werden, da z. B. eine mindestlohnbedingte Verkleinerung der Betriebsgröße durch eine steigende Zahl an Betrieben ausgeglichen werden könnte. Um einen besseren Einblick in die Gesamtwirkungen des Mindestlohns auf Branchenebene zu erhalten, berücksichtigen wir daher ergänzend die Wirkungen des Mindestlohns auf die Beschäftigung im Dachdeckerhandwerk auf der Ebene von Raumordnungsregionen.

Auf der Individualebene (vgl. Tab. 2) wird in einem ersten Ansatz die Veränderung in den Weiterbeschäftigungschancen der in den Geltungsbereich des Mindestlohns fallenden Beschäftigten des Dachdeckerhandwerks in der Zeit nach der Einführung des Mindestlohns (1997-2007) im Vergleich zu der Zeit vor dessen Einführung (19941996) ${ }^{7}$ mit der entsprechenden Veränderung der Weiterbeschäftigungschancen der theoretisch in den Geltungsbereich

\footnotetext{
${ }^{6} \mathrm{Da}$ die Daten auf individueller Ebene vorliegen, ermöglichen sie sowohl die Berechnung von Einstellungs- als auch Entlassungsraten auf Betriebsebene.

${ }^{7}$ Eine sukzessive Evaluation des Mindestlohns über die Zeit wird im Dachdeckerhandwerk dadurch erschwert, dass sich die Wirkungen der
} 
des Dachdecker-Mindestlohns fallenden Beschäftigten der Installationsbranche in einer multivariaten Differenz-vonDifferenzen-Schätzung (DvD) geschätzt. Dabei kontrolliert die Berücksichtigung einer Reihe von weiteren möglichen Determinanten der individuellen Beschäftigungschancen wie z. B. das Bildungsniveau mögliche Unterschiede in den beobachtbaren Charakteristika der Beschäftigten beider Branchen sowie eine mögliche Veränderung in der Zusammensetzung der Beschäftigten über die Zeit. Unter der Annahme, dass die so kontrollierte Entwicklung in der Installationsbranche die Entwicklung im Dachdeckerhandwerk ohne Mindestlohnregelung abbildet und es keine indirekten Auswirkungen des Mindestlohns im Dachdeckerhandwerk auf die Installationsbranche gibt, identifiziert eine entsprechende Differenz-von-Differenzen-Schätzung (DvD) den kausalen Effekt des Mindestlohns.

Demnach haben ostdeutsche Beschäftigte der Dachdeckerbranche nach der Mindestlohneinführung im Vergleich zu Beschäftigten der Installationsbranche eine um 0,7 Prozentpunkte gesunkene Wahrscheinlichkeit, im darauffolgenden Jahr in derselben Branche beschäftigt zu sein. Für Westdeutschland hingegen ergibt sich ein positiver Weiterbeschäftigungseffekt in Höhe von 1,5 Prozentpunkten.

Möglicherweise sind die Ergebnisse des Branchenvergleichs jedoch dadurch verzerrt, dass die Annahme paralleler Trends in den Branchen ohne Mindestlohnregelungen über den langen Zeitraum nicht zutrifft. Alternativ wird daher auch ein Vergleich der Weiterbeschäftigungschancen der von einem bindenden Mindestlohn betroffenen und nicht betroffenen Beschäftigten des Dachdeckerhandwerks vorgenommen. Da sich die Zusammensetzung der von einem bindenden Mindestlohn betroffenen Beschäftigten über die Zeit aufgrund der Anhebungen des Mindestlohns verändert, ist ein Vergleich dieser Personengruppen für die Jahre vor und nach der Mindestlohneinführung jedoch wenig sinnvoll. Stattdessen wird eine doppelte Differenzenbildung wiederum mittels der Installationsbranche vorgenommen, indem die Beschäftigten im Installationsgewerbe identifiziert werden, die im Falle einer Beschäftigung in der Dachdeckerbranche von einem bindenden Mindestlohn betroffen wären. ${ }^{8}$ Dies wird möglich, indem das für die Dachdecker-

verschiedenen Mindestlohnerhöhungen vermutlich überlagern. Versuche der Evaluation einzelner Mindestlohnanhebungen ergaben teilweise sehr unklare Wirkungen, so dass der Fokus der Analysen auf einem Vergleich der Zeit vor und nach der Mindestlohneinführung liegt (vgl. Aretz et al. 2011).

${ }^{8}$ Da für die BA-Daten keine exakte Einteilung der Beschäftigten in Beschäftigte mit und ohne bindenden ML vorgenommen werden kann (vgl. Anhang B), geht die sich aus der Imputation ergebende Betroffenheitswahrscheinlichkeit als Gewicht in die Schätzung ein. Ein Individuum, welches mit einer Wahrscheinlichkeit von 80 Prozent einem bindenden ML unterliegt, wird in der Schätzung somit zu 80 Prozent zur Gruppe der Betroffenen gezählt und zu 20 Prozent zu der Gruppe der nicht Betroffenen. Dadurch wird die Unschärfe in der Imputation des Stundenlohns bestmöglich in der Schätzung berücksichtigt. branche entwickelte Imputationsmodell (vgl. Anhang B) auf die Installationsbranche angewendet wird, so dass eine kontrafaktische Wahrscheinlichkeit berechnet werden kann, von einem bindenden Mindestlohn betroffen zu sein.

Dieser Ansatz beruht auf der Annahme, dass Beschäftigten im Installationsgewerbe am ehesten mit denjenigen Beschäftigten im Dachdeckerhandwerk vergleichbar sind, die aufgrund ihrer Charakteristika im Falle einer Beschäftigung in der Dachdeckerbranche in ähnlicher Weise vom Mindestlohn betroffen wären. Durch eine doppelte Differenz der von einem bindenden Mindestlohn betroffenen und der nicht betroffenen Beschäftigten in beiden Branchen lässt sich zudem für abweichende Trends zwischen den Branchen kontrollieren. Dies setzt jedoch voraus, dass sich die Beschäftigung von Personen mit und ohne bindenden Mindestlohn ohne Mindestlohnregelung in ähnlicher Weise entwickelt hätte und keine indirekten Auswirkungen des Mindestlohns auf die nicht von einem bindenden Mindestlohn betroffenen Beschäftigten in den beiden Branchen bestehen.

Im Ergebnis (Zeile 2 in Tab. 2) haben westdeutsche Beschäftigte des Dachdeckerhandwerks mit einem bindenden Mindestlohn eine um sechs Prozentpunkte reduzierte Wahrscheinlichkeit, im darauffolgenden Jahr weiterhin in der Branche beschäftigt zu sein. Bei einer gegebenen Betroffenheitswahrscheinlichkeit von durchschnittlich 5,5\% zwischen 1997 und 2007 impliziert dies eine um 0,3 Prozentpunkte reduzierte Weiterbeschäftigungswahrscheinlichkeit auf Branchenebene (analog zu Ansatz 1). ${ }^{9}$ Für Ostdeutschland reduziert sich die Wahrscheinlichkeit einer Weiterbeschäftigung in der Dachdeckerbranche für die von einem bindenden Mindestlohn betroffenen Beschäftigten um 2,8 Prozentpunkte. Dies entspräche bei einem durchschnittlichen Anteil der betroffenen Personen von etwa $20 \%$ einer um etwa 0,6 Prozentpunkte reduzierten Weiterbeschäftigungswahrscheinlichkeit auf Branchenebene. Während beide Ansätze für Ostdeutschland somit zu sehr vergleichbaren und negativen Auswirkungen auf die Weiterbeschäftigungschancen kommen, deutet die Abweichung beider Ansätze für Westdeutschland hingegen darauf hin, dass die zugrundeliegenden Annahmen teilweise verletzt sind. So könnten die Ergebnisse ein Hinweis darauf sein, dass mindestlohnbedingt weniger qualifizierte durch gut qualifizierte Beschäftigte substituiert werden und somit auch Beschäftigte im Dachdeckerhandwerk ohne einen bindenden Mindestlohn indirekt vom Mindestlohn betroffen sind. Die Ergebnisse für Westdeutschland können somit nur bedingt als verlässlich eingestuft werden. Zudem können die Ergebnisse beider Ansätze verzerrt sein, wenn ein Betrieb mindestlohnbedingt vor allem die Beschäftigten mit einer geringen Motivation oder Fähigkeit entlässt, da diese unbeobachteten Faktoren

\footnotetext{
${ }^{9}$ Für eine ausführliche Beschreibung der Ermittlung der Betroffenheitswahrscheinlichkeit in den BA-Daten siehe Anhang B.
} 
Tab. 2 Mindestlohnwirkungen auf die Weiterbeschäftigungswahrscheinlichkeit der Beschäftigten der Dachdeckerbranche, verschiedene DvD-Schätzungen auf Basis der BA-Daten

\begin{tabular}{|c|c|c|c|c|}
\hline & Abhängige Variable (Y) & Treatmentgruppe/Kontrollgruppe & DvD-Ansatz & $\begin{array}{l}\text { Marginaler Effekt in } \\
\text { Prozentpunkten }^{\mathrm{a}}\end{array}$ \\
\hline (1) & $\begin{array}{l}\text { Indikatorvariable für eine im } \\
\text { nächsten Jahr fortgesetzte } \\
\text { Beschäftigung in der Branche }\end{array}$ & $\begin{array}{l}\text { Gewerbliche abhängig } \\
\text { Beschäftigte im Dachdecker- } \\
\text { handwerk/Installationsgewerbe }\end{array}$ & $\begin{array}{l}\text { Vergleich der Differenz vor ML } \\
\text { (1994-1996) und nach ML (1997-2007) }\end{array}$ & $\begin{array}{l}\text { West: } 1,5^{* * *} \\
\text { Ost: }-0,7^{* * *}\end{array}$ \\
\hline (2) & $\begin{array}{l}\text { Indikatorvariable für eine im } \\
\text { nächsten Jahr fortgesetzte } \\
\text { Beschäftigung in der Branche }\end{array}$ & $\begin{array}{l}\text { Beschäftigte ohne bindenden } \\
\text { Mindestlohn/Beschäftigte mit } \\
\text { bindendem Mindestlohn im } \\
\text { Dachdeckerhandwerk }\end{array}$ & $\begin{array}{l}\text { Vergleich der Differenz mit der Differenz } \\
\text { zwischen Beschäftigten im } \\
\text { Installationsgewerbe mit und ohne } \\
\text { kontrafaktisch bindenden ML, } \\
\text { 1997-2007 }\end{array}$ & $\begin{array}{l}\text { West: }-6,0^{* * *} \\
\text { Ost: }-2,8^{* * *}\end{array}$ \\
\hline
\end{tabular}

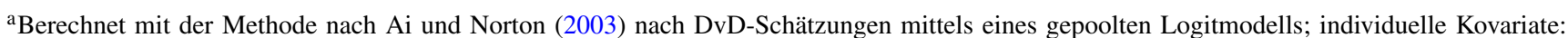
Alter, Geschlecht, Bildungsabschluss sowie Teilzeitdummy; betriebliche Kovariate: Anteil der Facharbeiter/Innen und ungelernten Arbeiter/Innen, durchschnittliches Belegschaftsalter sowie in drei Gruppen unterteilte Unternehmensgröße; den Schätzungen liegen im Westen ca. 1,2 Millionen und im Osten ca. 530.000 Beobachtungen aus den BA-Daten zugrunde

Tab. 3 Mindestlohnwirkungen auf den Beschäftigungsumfang und den Fachkräfteanteil auf Betriebsebene, verschiedene DvD-Ansätze auf Basis der BA-Daten

\begin{tabular}{|c|c|c|c|c|}
\hline & Abhängige Variable & Treatmentgruppe/Kontrollgruppe & DvD-Ansatz & Effekt $^{\mathrm{a}}$ \\
\hline (1) & $\begin{array}{l}\text { Zahl der Beschäftigten } \\
\text { pro Betrieb }\end{array}$ & \multirow[t]{2}{*}{ Dachdeckerbetriebe/Installationsbetriebe } & \multirow[t]{2}{*}{$\begin{array}{l}\text { Vergleich der Differenz vor ML } \\
\text { (1994-1996) und nach ML (1997-2007) }\end{array}$} & $\begin{array}{l}\text { West: }-8,0^{* * *} \\
\text { Ost: }-0,5\end{array}$ \\
\hline (2) & Anteil Facharbeitskräfte & & & $\begin{array}{l}\text { West: } 6,1^{* * *} \\
\text { Ost: } 5,7^{* * *}\end{array}$ \\
\hline (3) & $\begin{array}{l}\text { Zahl der Beschäftigten } \\
\text { pro Betrieb }\end{array}$ & \multirow{2}{*}{$\begin{array}{l}\text { Vom ML betroffene Dachdeckerbetriebe/ } \\
\text { vom ML nicht betroffene } \\
\text { Dachdeckerbetriebe }\end{array}$} & \multirow{2}{*}{$\begin{array}{l}\text { Vergleich der Differenz mit der Differenz } \\
\text { zwischen kontrafaktisch betroffenen und } \\
\text { nicht betroffenen Installationsbetrieben } \\
\text { im Zeitraum 1997-2007 }\end{array}$} & $\begin{array}{l}\text { West: }-3,6^{* * *} \\
\text { Ost: }-8,1^{* * *}\end{array}$ \\
\hline (4) & Anteil Facharbeitskräfte & & & $\begin{array}{l}\text { West: } 2,3^{* * *} \\
\text { Ost: } 2,5^{* * *}\end{array}$ \\
\hline
\end{tabular}

${ }^{a}$ Lineare DvD-Schätzungen mit betriebsspezifischen fixen Effekten; Kovariate: durchschnittliches Alter der Beschäftigten, Anteil ganzjährig bzw. zu Jahresbeginn Beschäftigter, durchschnittliche Betriebszugehörigkeit der Beschäftigten, durchschnittliche Anzahl der Unterbrechungen von laufenden Beschäftigungsverhältnissen, Alter des Betriebs sowie verschiedene Nachfrageindikatoren wie z. B. Summe der Marktein- und -austritte im Kreis und Marktanteil des größten Unternehmens im Kreis; den Schätzungen liegen im Westen ca. 200.000 und im Osten ca. 73.000 Betriebsbeobachtungen aus den BA-Daten zugrunde. Effekte für (1) und (3) in Prozent; Effekte für (2) und (4) in Prozentpunkten

bislang in den Schätzmodellen nicht berücksichtigt werden. Hier besteht noch weiterer Forschungsbedarf.

Unter Verwendung derselben Kontrollgruppenansätze betrachten wir auf der Betriebsebene den Umfang der gewerblichen Beschäftigung sowie den Anteil der Fachkräfte auf Betriebsebene, vgl. Tab. 3. Während die betriebliche Beschäftigung im Vergleich zu den vorangegangenen Analysen zusätzlich zum Entlassungsverhalten auch das Einstellungsverhalten der Betriebe abbildet, soll der Fachkräfteanteil einen Einblick in die möglicherweise aufgrund von Substitutionseffekten veränderte Beschäftigungsstruktur geben. Die linearen Schätzungen erfolgen zudem unter Berücksichtigung betrieblicher Charakteristika sowie betriebsspezifischer fixer Effekte und kontrollieren somit für beobachtbare und unbeobachtete, zeitkonstante Faktoren auf der Betriebsebene. Dies ist deshalb besonders wichtig, da mindestlohnbedingte Marktaustritte gering produktiver Betriebe die Ergebnisse andernfalls verzerren könnten.
Die entsprechenden Auswirkungen des Mindestlohns werden in dem ersten Kontrollgruppenansatz (vgl. Zeilen 1 und 2, Tab. 3) im Rahmen eines Vergleichs von Dachdeckerund Installationsbetrieben vor und nach der Mindestlohneinführung bestimmt. Für den zweiten Kontrollgruppenansatz (vgl. Zeilen 3 und 4, Tab. 3) wird hingegen die Differenz in den Zielgrößen für die betroffenen im Vergleich zu den nicht betroffenen Dachdeckerbetrieben in der Zeit nach der Mindestlohneinführung mit der kontrafaktischen Differenz zwischen betroffenen und nicht betroffenen Installationsbetrieben verglichen. Ein Betrieb gilt dabei als vom Mindestlohn betroffen, wenn die durchschnittliche - im Falle der Installationsbetriebe kontrafaktische - Betroffenheitswahrscheinlichkeit der Beschäftigten des Betriebs über $5 \%$ liegt.

Im Branchenvergleich zeigt sich für Westdeutschland, dass die durchschnittliche Betriebsgröße im Dachdeckerhandwerk gegenüber dem Installationsgewerbe mindestlohnbedingt um $8 \%$ gesunken ist, während der Rück- 
gang der durchschnittlichen Betriebsgröße in Ostdeutschland nicht signifikant ist. Gleichzeitig stieg der durchschnittliche Fachkräfteanteil auf Betriebsebene im Branchenvergleich in beiden Landeshälften um etwa sechs Prozentpunkte. Eine Verschiebung der Beschäftigtenstruktur hin zu höher qualifizierten Beschäftigten wird auch durch den zweiten Kontrollgruppenansatz - wenn auch in geringerem Umfang - bestätigt. Der Beschäftigungsrückgang seit Mitte der 1990er Jahre hat demnach - auch unterstützt durch den Mindestlohn - stärker die gering Qualifizierten der Branche betroffen.

Umgerechnet auf Branchenebene ergibt sich nach dem zweiten Ansatz (Zeile 3) bei einer durchschnittlichen Betroffenheit der Betriebe in Westdeutschland (Ostdeutschland) in Höhe von $8 \%$ (26\%) eine durchschnittliche Reduktion der Beschäftigung je Betrieb um 0,3 \% (2,1\%). Der geschätzte Rückgang der durchschnittlichen Betriebsgröße fällt in Ostdeutschland somit höher und in Westdeutschland niedriger aus als im reinen Branchenvergleich. Diese Abweichungen sprechen dafür, dass der Branchenvergleich in Zeile (1) beispielsweise aufgrund abweichender Branchentrends verzerrt ist. Abweichende Trends können durch die Differenzenbildung zwischen betroffenen und nicht betroffenen Betrieben innerhalb jeder Branche in Zeile (3) jedoch berücksichtigt werden, so dass diese Ergebnisse eine höhere Validität aufweisen sollten.

Eine solche Reduktion der durchschnittlichen Beschäftigung auf Betriebsebene bedeutet jedoch nicht zwingend auch einen Rückgang der Gesamtbeschäftigung, da es gleichzeitig zu einer Zunahme der Zahl der Betriebe gekommen sein kann. Tatsächlich lässt sich deskriptiv beobachten, dass die Zahl der Ein-Personen-Unternehmen stark zugenommen hat. Um somit alle möglichen Wirkungsmechanismen (Einstellungs- und Separationsverhalten der bereits existierenden Betriebe, Betriebsgründungen/-schließungen, Spillovereffekte zwischen betroffenen und nicht betroffenen Betrieben) zu berücksichtigen und die Wirkungen auf die Gesamtbeschäftigung zu identifizieren, ist eine Analyse auf Marktebene erforderlich, indem die Gesamtbeschäftigung auf der Ebene der Raumordnungsregionen betrachtet wird. Dafür nutzen wir die regionale Variation in dem Anteil der vom Mindestlohn betroffenen Beschäftigten sowie die regionale Variation im Kaitz-Index unter der für das Dachdeckerhandwerk plausiblen Annahme, dass es sich bei diesen regionalen Märkten um relativ abgeschlossene Märkte handelt. Die Schätzungen auf Basis der BA- und der LAK-Daten erfolgen unter Berücksichtigung regionaler fixer Effekte, so dass die Veränderung in der regionalen Betroffenheit vom Mindestlohn bzw. des Kaitz-Indexes den Mindestlohneffekt identifiziert. Gleichzeitig werden in dem multivariaten Schätzansatz regionale Marktunterschiede durch die Anzahl der Baugenehmigungen und Fertigstellungen, die Anzahl der Gebäude, den Sanierungsindex, die
Altersstruktur der Gebäude, die neu installierte Leistung an Photovoltaik-Anlagen sowie die Zahl der Personen in der Region und die Kaufkraft gemessen am Bruttoinlandsprodukt pro Kopf berücksichtigt.

Die Effekte auf die Gesamtbeschäftigung in der Branche auf regionaler Ebene zeigen keine signifikanten Auswirkungen des Mindestlohns. Dieses Ergebnis spricht entweder für einen Nulleffekt des Mindestlohns auf die Gesamtbeschäftigung oder für eine zu geringe Identifikationskraft des gewählten Schätzansatzes. Letzteres könnte der Fall sein, da die regionale Betroffenheit vom Mindestlohn nicht aufgrund unterschiedlicher Mindestlohnhöhen, sondern lediglich aufgrund unterschiedlicher Marktstrukturen variiert. Unter weitgehender Kontrolle der regionalen Marktstrukturen ist die Identifikationskraft des Schätzansatzes somit möglicherweise beschränkt. In diesem Fall sprechen die vorangegangenen Analysen zumindest für Ostdeutschland für einen mindestlohnbedingten Beschäftigungsrückgang.

Wertet man die regionalen Analysen hingegen als verlässliche Evidenz für einen wahrscheinlichen Nulleffekt des Mindestlohns auf die Gesamtbeschäftigung, steht dieses Ergebnis in einem vermeintlichen Widerspruch zu den vorangegangenen Analysen. Es ist jedoch durchaus denkbar, dass es mindestlohnbedingt zu Umstrukturierungen kam und der Mindestlohn die Beschäftigungschancen der von einem bindenden Mindestlohn betroffenen Beschäftigten verschlechtert sowie in betroffenen Betrieben zu einem Beschäftigungsrückgang beigetragen hat. Der nicht vorhandene Effekt auf der Ebene des (regionalen) Marktes deutet jedoch darauf hin, dass diese Wirkungen sich nicht in Nettoverlusten in der Branche niederschlugen, da nicht betroffene Fachkräfte und/oder andere Betriebe der Branche indirekt profitieren und Beschäftigungsverluste in Teilen der Branche ausgeglichen haben.

\subsection{Arbeitnehmerschutz}

Die Auswirkungen des Mindestlohns auf den Arbeitnehmerschutz werden anhand der Entlohnungs- und Arbeitsbedingungen in der Branche untersucht. Dabei ist vor allem fraglich, ob sich mindestlohnbedingt steigende Stundenlöhne auch in steigende Einkommen übersetzen. Dies hängt nicht zuletzt vom Arbeitsumfang ab, da steigende Stundenlöhne bei einer gleichzeitigen Reduktion der Arbeitszeit mit sinkenden Lohneinkommen einhergehen können. Die Jahreslohneinkommen in den LAK-Daten sind jedoch durch diverse Sonderzahlungen, wie z. B. das 13. Monatsgehalt, beeinflusst. Stattdessen betrachten wir daher die Bruttotagesentgelte. Zwar berücksichtigen diese keine Einkommenseffekte, die durch verkürzte Jahresarbeitszeiten z. B. aufgrund saisonaler Entlassungen entstehen; sie bilden jedoch den Einfluss der durchschnittlichen täglichen Arbeitszeit auf das Lohneinkommen ab. 
Tab. 4 Bruttostundenlöhne, Tagesarbeitszeiten und Bruttotagesentgelte zwischen 1995 und 2010 nach Dezilen der Stundenlohnverteilung, Juni-Monatsangaben der LAK-Daten

\begin{tabular}{|c|c|c|c|c|c|c|}
\hline & \multicolumn{2}{|c|}{ Bruttostundenlöhne } & \multicolumn{2}{|c|}{ Arbeitsstunden pro Arbeitstag } & \multicolumn{2}{|c|}{ Bruttotagesentgelte } \\
\hline & $\begin{array}{l}1995 \\
(\text { in €) }\end{array}$ & $\begin{array}{l}\Delta 2010 / 1995 \\
\text { (in \%) }\end{array}$ & $\begin{array}{l}1995 \\
\text { (in h) }\end{array}$ & $\begin{array}{l}\Delta 2010 / 1995 \\
\text { (in \%) }\end{array}$ & $\begin{array}{l}1995 \\
\text { (in €) }\end{array}$ & $\begin{array}{l}\Delta 2010 / 1995 \\
\text { (in \%) }\end{array}$ \\
\hline \multicolumn{7}{|l|}{ Ostdeutschland } \\
\hline \multicolumn{7}{|l|}{ Dezil1 } \\
\hline Perzentile 1 bis 5 & 6,0 & 68,2 & 7,0 & $-12,8$ & 44,7 & 44,9 \\
\hline Perzentile 5 bis 10 & 6,9 & 53,6 & 7,6 & $-8,4$ & 55,0 & 37,8 \\
\hline Dezil 2 & 7,5 & 40,8 & 7,7 & $-9,7$ & 60,0 & 26,0 \\
\hline Dezil 3 & 8,2 & 30,1 & 7,7 & $-9,9$ & 64,8 & 16,5 \\
\hline Dezil 4 & 8,7 & 22,1 & 7,7 & $-23,7$ & 68,7 & $-6,8$ \\
\hline Dezil 5 & 9,1 & 16,3 & 7,7 & $-19,9$ & 72,0 & $-7,3$ \\
\hline Dezil 6 & 9,6 & 12,5 & 7,6 & $-10,3$ & 75,6 & 0,8 \\
\hline Dezil 7 & 10,1 & 9,0 & 7,6 & $-11,1$ & 79,2 & $-3,0$ \\
\hline Dezil 8 & 10,8 & 5,9 & 7,6 & $-9,0$ & 84,0 & $-3,3$ \\
\hline Dezil 9 & 11,7 & 3,3 & 7,6 & $-8,1$ & 90,4 & $-5,5$ \\
\hline Dezil 10 & 13,7 & 0,2 & 7,5 & $-7,0$ & 100,8 & $-4,9$ \\
\hline Median & 9,4 & 14,5 & 7,6 & $-8,9$ & 73,5 & 9,4 \\
\hline \multicolumn{7}{|l|}{ Westdeutschland } \\
\hline \multicolumn{7}{|l|}{ Dezil1 } \\
\hline Perzentile 1 bis 5 & 6,8 & 44,6 & 3,0 & 43,0 & 22,2 & 102,1 \\
\hline Perzentile 5 bis 10 & 8,7 & 22,9 & 6,5 & $-32,6$ & 60,3 & $-18,9$ \\
\hline Dezil 2 & 9,7 & 18,4 & 7,1 & $-13,3$ & 72,7 & 0,5 \\
\hline Dezil 3 & 10,5 & 20,3 & 7,3 & $-8,0$ & 80,5 & 8,4 \\
\hline Dezil 4 & 11,2 & 19,0 & 7,4 & $-4,5$ & 87,4 & 11,3 \\
\hline Dezil 5 & 11,9 & 18,3 & 7,4 & $-4,0$ & 92,1 & 11,3 \\
\hline Dezil 6 & 12,3 & 18,6 & 7,3 & $-2,7$ & 95,7 & 11,9 \\
\hline Dezil 7 & 12,7 & 20,3 & 7,3 & $-4,5$ & 98,3 & 12,1 \\
\hline Dezil 8 & 13,1 & 21,2 & 7,4 & $-3,8$ & 101,5 & 14,2 \\
\hline Dezil 9 & 13,8 & 20,6 & 7,4 & $-4,4$ & 105,5 & 14,8 \\
\hline Dezil 10 & 16,1 & 14,3 & 7,4 & $-3,2$ & 117,0 & 12,6 \\
\hline Median & 12,1 & 17,7 & 7,2 & $-3,2$ & 92,6 & 13,4 \\
\hline
\end{tabular}

Tabelle 4 weist die in den LAK Daten beobachteten prozentualen Veränderungen in den Stundenlöhnen, den Arbeitsstunden pro Arbeitstag und den daraus resultierenden Bruttotagesentgelten zwischen 1995 und 2010 entlang der Stundenlohnverteilung aus. Zum einen bestätigt sich die bereits in Abschn. 3 diskutierte Entwicklung, dass die von einem bindenden Mindestlohn betroffenen unteren Dezile der Lohnverteilung - in Ostdeutschland bis zum 6. Dezil, in Westdeutschland bis zum 1. Dezil (vgl. Tab. 1) - stärkere Stundenlohnzuwächse verzeichnen, als dies in den oberen Dezilen der Fall ist. Dies trifft insbesondere auf Ostdeutschland zu, da sich die Stundenlöhne am oberen Rand der Lohnverteilung in dem Zeitraum nominal kaum verändert haben.

Auch trotz der unvollständigen Einhaltung des Mindestlohns (vgl. Tab. 1) führt der Mindestlohn somit zumindest teilweise zu gewünschten Stundenlohnzuwächsen. Diese übersetzen sich jedoch nicht Eins zu Eins in entsprechen- de Einkommenszuwächse. So steigen die (nominalen) Bruttotagesentgelte in Ostdeutschland lediglich bis zum dritten Dezil der Lohnverteilung; alle anderen Dezile erfahren sogar einen nominalen Einkommensrückgang. Dies spiegelt die Veränderungen in den täglichen Arbeitszeiten wider. Zwar sank die Arbeitszeit für alle Dezile der Lohnverteilung; vor allem die Arbeitszeit der mittleren Dezile hat sich jedoch reduziert, so dass hier Stundenlohnzuwächse durch verringerte Arbeitszeiten überkompensiert werden. Auch in Westdeutschland reduzierten sich die täglichen Arbeitszeiten für nahezu alle Beschäftigten. Überdurchschnittliche Arbeitszeitverkürzungen finden sich vor allem in den Perzentilen direkt oberhalb der vom Mindestlohn betroffenen Perzentile, während die unteren fünf Perzentile sogar stark an Arbeitszeit zulegten. Hierbei ist jedoch $\mathrm{zu}$ beachten, dass es sich um rein deskriptive Veränderungen handelt, die u. a. dadurch zustande kommen können, dass mindestlohnbedingt 
Abb. 5 Prozentuale

Veränderung der

Bruttotageseinkommen im

Dachdecker- im Vergleich zum

Installationshandwerk nach

Einkommensdezilen, BA-Daten

1995-2008. Anm.: Separate

DvD-Schätzungen des

logarithmierten

Bruttotagesentgelts mit der

Installationsbranche als

Kontrollgruppe für jedes

Einkommensdezil; gepunktete

Balken sind auf dem

$5 \%$-Signifikanznvieau

insignifikant
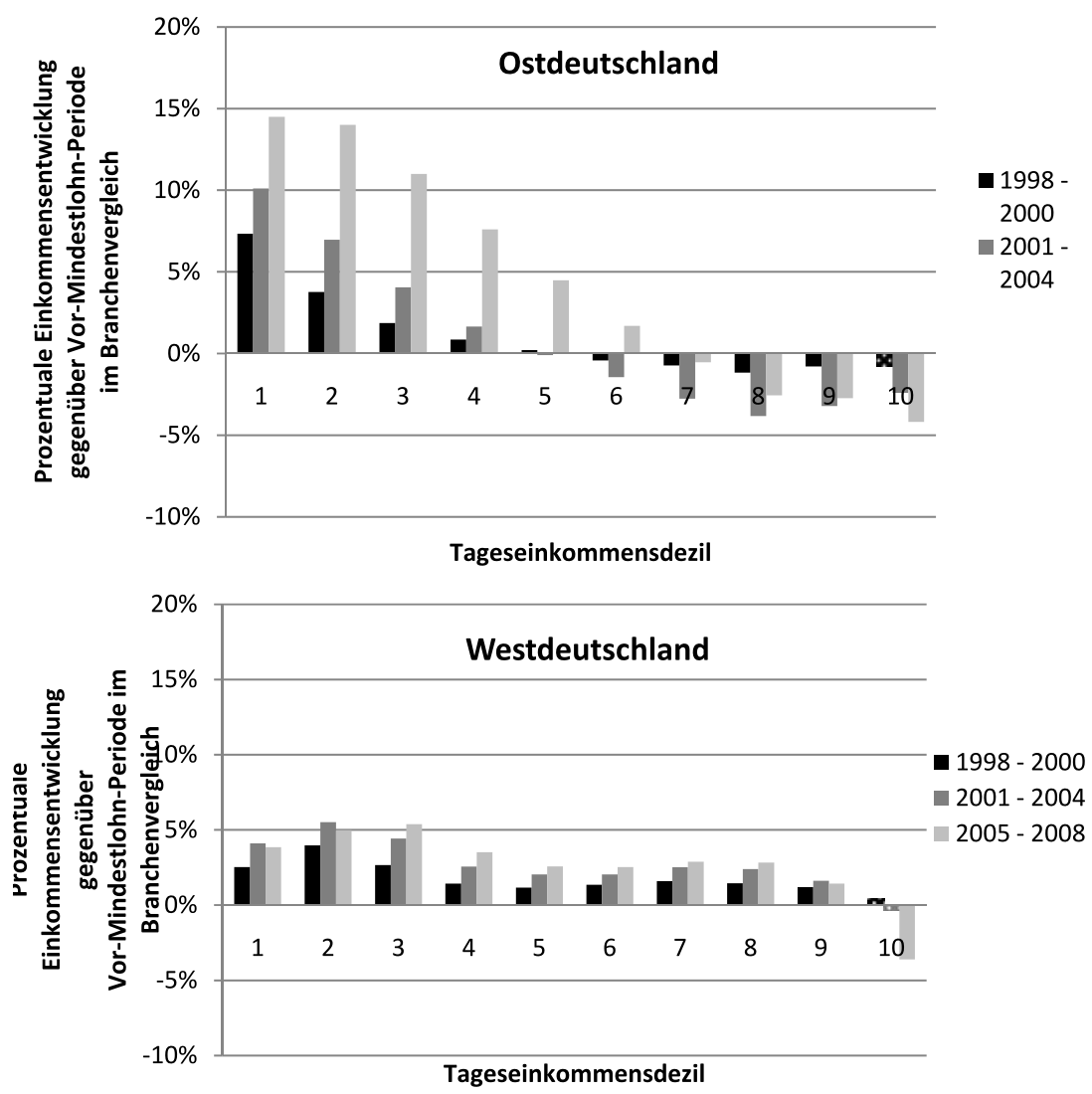

vor allem die von einem bindenden Mindestlohn betroffenen Beschäftigten mit sehr geringen Arbeitszeiten entlassen wurden. Im Ergebnis haben die weiterhin Beschäftigten in den unteren fünf Perzentilen einen deutlichen Zuwachs der nominalen Bruttotagesentgelte zu verzeichnen, während die oberen Dezile in etwa einen dem Durchschnitt entsprechenden Einkommenszuwachs erfahren haben.

Die beobachteten Veränderungen können jedoch nicht unbedingt als ein kausaler Effekt des Mindestlohns interpretiert werden, da diese Veränderungen auch auf allgemeine konjunkturelle Einflüsse zurückgehen könnten. Zudem kann sich die Zusammensetzung der Beschäftigten über die Zeit verändern, so dass die Vergleichbarkeit der Beschäftigten in den Dezilen nicht gewährleistet ist. Aus diesem Grund untersuchen wir mit Hilfe der BA-Daten die Einkommensentwicklung im Kontext eines multivariaten Schätzansatzes, der das logarithmierte Bruttotagesentgelt zwischen der Dachdecker- und der Installationsbranche in der Zeit nach der Mindestlohneinführung im Vergleich zu der Zeit davor für jedes Einkommensdezil getrennt vergleicht. Zudem werden nach der Mindestlohneinführung drei verschiedene Phasen unterteilt, die jeweils mit der Branche interagiert werden und somit zusätzliche heterogene Wirkungen im Zeitablauf zulassen. Diese zeitliche Aufschlüsselung erlaubt es, die Wirkung des Mindestlohns besser in einen Zusammenhang zum steigenden Mindestlohnniveau zu setzen, da im
Laufe der Zeit vor allem in Ostdeutschland ein wachsender Anteil der Beschäftigten einem bindenden Mindestlohn unterlag. Das in Abb. 5 dargestellte Ergebnis zeigt für jedes Einkommensdezil und jede der drei Perioden den um beobachtbare Charakteristika ${ }^{10}$ bereinigten prozentualen Anstieg des Bruttotagesentgelts gegenüber der Vormindestlohnperiode im Vergleich zur Installationsbranche.

Für Ostdeutschland zeigt Abb. 5, dass die untersten Einkommensdezile bereits in der Einführungsphase deutliche Einkommenszuwächse im Vergleich zur Installationsbranche und Vorperiode erfuhren. Von dieser Entwicklung konnten mit zunehmendem Betrachtungszeitraum - und damit einem steigenden Anteil an betroffenen Beschäftigten - systematisch auch höhere Einkommensdezile profitieren. Dagegen mussten besser verdienende Dachdecker/Innen im Vergleich zur Installationsbranche Einkommensverluste hinnehmen. Zieht man die Erkenntnisse aus den LAK-Daten hinzu, ist diese Entwicklung vermutlich das Resultat von weitgehend stagnierenden Nominallöhnen für die besserverdienenden Beschäftigten der Branche und einer in den letzten Jahren insgesamt reduzierten Arbeitszeit. Diese sinken-

\footnotetext{
${ }^{10}$ Als Kovariate gehen in das Modell das Alter (bis zur vierten Potenz), Dummies für das Geschlecht, eine Ganzjahrestätigkeit, Teilzeittätigkeit und Fachkraft sowie Dummies für die 97 Raumordnungsregionen ein.
} 
den Arbeitskosten für die besserverdienenden Beschäftigten der Branche konnten somit die steigenden Arbeitskosten bei den schlechter Verdienenden teilweise kompensieren. Ergebnis ist das weitgehende Verschwinden einer Lohndifferenzierung in Ostdeutschland (vgl. auch Abb. 4). In Gesprächen mit Verbandsvertretern wurde diese Entwicklung mehrfach bestätigt. Auf die Rückfrage hin, wieso Unternehmen in der Vergangenheit ihre qualifizierten Beschäftigten zu solchen Konditionen trotz des großen Lohnunterschieds zwischen Ost- und Westdeutschland halten konnten, wurde wiederholt auf die hohe Bindung der Beschäftigten an Betriebe und die geringe regionale Mobilität verwiesen. Für die ostdeutschen Betriebe würde es aktuell jedoch zunehmend schwieriger, Fachkräfte zu finden und zu halten, da sich mittlerweile auch in Folge stark gesunkener Azubizahlen ein Fachkräftemangel in der Branche abzeichnet. In den Betrieben müsste bei der Entlohnung der Beschäftigten daher bald ein Umdenken einsetzen.

Für Westdeutschland zeigt Abb. 5 für alle außer dem obersten Dezil Einkommenszuwächse im Vergleich zur Vorperiode und zur Installationsbranche. Auffällig ist, dass die drei unteren Dezile in Westdeutschland eine überdurchschnittliche Einkommensverbesserung im Vergleich zur Referenzgruppe erzielen konnten, obwohl nur maximal $15 \%$ der Beschäftigten vom Mindestlohn direkt betroffen waren. Dies kann ein Indiz dafür sein, dass sich die Nachfrage nach Arbeitskräften mit einem Verdienst knapp oberhalb der Lohnuntergrenze mindestlohnbedingt erhöht hat. Überraschend ist, dass die positiven Einkommenswirkungen bis zum 9. Dezil reichen. Dies deutet möglicherweise daraufhin, dass das Dachdeckerhandwerk in Westdeutschland eine auch vom Mindestlohn unabhängige, insgesamt günstigere Entwicklung als das Installationsgewerbe genommen hat und die Vergleichbarkeit der beiden Branchen somit nicht gänzlich gegeben ist. Möglich ist jedoch auch, dass die aufgrund des Mindestlohns reduzierte Wettbewerbsverzerrung zwischen Ost- und Westdeutschland hier zu einer positiven Einkommensentwicklung beigetragen hat, da es unter einem reduzierten Preiswettbewerb eher möglich ist, höhere Preise und Einkommen durchzusetzen. Die Bedeutung des Mindestlohns zur Reduktion von Wettbewerbsverzerrungen und Begrenzung eines Preiswettbewerbs wurde wiederholt durch Verbandsvertreter im Rahmen von Expertengesprächen betont.

Die Wirkungen des Mindestlohns können jedoch über die Entlohnungs- und Einkommensbedingungen hinausgehen. Falls der Wettbewerb auf dem Arbeitsmarkt laut Einschätzungen von Branchenvertretern in Ostdeutschland zumindest teilweise beschränkt ist, könnte es beispielsweise zu einer Verschlechterung von Arbeitsbedingungen (z. B. der Vergütung von Arbeitsstunden, Maßnahmen zur Arbeitssicherheit) kommen, um an anderer Stelle Kosten einzusparen. Da entsprechende Indikatoren in den vor- handenen Sekundärdaten fehlen, wurden zusätzliche Indikatoren zum Arbeitnehmerschutz im Rahmen der CATIUnternehmensbefragung sowohl für Dachdecker- als auch Installationsbetriebe (siehe Anhang A) erhoben. Dies betraf zum einen die Frage nach einer Entlohnung zum Tarifvertrag, das im Unternehmen angewandte Arbeitszeit- und Überstundenmodell, den Krankenstand des Unternehmens sowie das Ausmaß der prekären Beschäftigung in Form der nicht dauerhaft beschäftigten Randbelegschaft. Die Analysen konnten keine mindestlohnbedingten Veränderungen in den untersuchten Zielgrößen finden. Dies bedeutet entweder, dass es hier keine Auswirkungen gegeben hat oder dass die Datenlage nicht ausreichte, Wirkungen des Mindestlohns nachzuweisen.

\subsection{Effekte auf die Wettbewerbssituation}

Die Auswirkungen des Mindestlohns auf den Wettbewerb im Dachdeckerhandwerk werden anhand von fünf Wettbewerbsindikatoren analysiert, nämlich der Profitabilität der im Markt aktiven Unternehmen, der Marktaustritte, der Markteintritte, der Anzahl der im Markt aktiven Unternehmen (als Maß für die Angebotskonzentration bei gegebener Nachfrage) sowie der Preise für Dachdeckerleistungen. $\mathrm{Zu}$ den einzelnen Indikatoren liegen Messwerte auf unterschiedlichen Aggregationsniveaus vor: Profitabilität und Marktaustrittswahrscheinlichkeit können auf Unternehmensebene gemessen werden, Angaben zu Marktzutritten und zur Anzahl der im Markt aktiven Unternehmen liegen auf der Ebene regional abgegrenzter Marktgebiete vor, während Güterpreise nur für Deutschland insgesamt (auf Basis der Preisindizes des Baugewerbes) gemessen werden können. Daraus ergeben sich jeweils spezifische empirische Untersuchungsansätze. Gemeinsam ist allen Ansätzen, dass neben der Mindestlohnvariable noch weitere Bestimmungsfaktoren des Wettbewerbs abgebildet werden, um spezifische Produktions- und Nachfragebedingungen der Branchen zu berücksichtigen. ${ }^{11}$ Mindestlohneffekte werden daher über multivariate Erklärungsmodelle, die in einen DvD-Rahmen eingebettet sind, empirisch geschätzt. Dabei werden neben dem Mindestlohn auch viele weitere Einflussgrößen von Wettbewerbsvariablen berücksichtigt. Als Kontrollbranche dient das Installationsgewerbe. Der Untersuchungszeitraum

\footnotetext{
${ }^{11}$ Es sind dies unternehmensspezifische (Alter, Größe, Rechtsform, Qualifikation des Inhabers), nachfrageseitige (Neubautätigkeit, Sanierungsbedarf, Zubau an Photovoltaikanlagen auf Dächern, jeweils bezogen auf den regionalen Absatzmarkt), faktor- und bezugsmarktseitige (Arbeitskostenentwicklung unabhängig vom Mindestlohn, Inputpreisentwicklung) und wettbewerbsspezifische (Marktanteil des Unternehmens, Anzahl der Wettbewerber im regionalen Absatzmarkt, Distanz zum nächstliegenden Wettbewerber) Variablen. Daten zu diesen Variablen werden aus dem MUP, der amtlichen Statistik, der BA sowie einer Sonderauswertung Forschungsstelle für Energiewirtschaft (Zubau an Photovoltaikanlagen auf Dächern) gewonnen.
} 
umfasst die Jahre 1996 bis 2010 und schließt somit auch einen Zeitraum vor Mindestlohneinführung sowie die Perioden der kurzzeitigen Unterbrechung in den Jahren 2000/01 und 2002/03 mit ein.

Daten zu Profitabilität, Marktaustritten, Markteintritten und Unternehmensbestand werden aus dem MUP gewonnen (siehe Anhang A). Die Profitabilität wird über die Bonitätseinstufung der Unternehmen approximiert, die von der Kreditauskunftei Creditreform vorgenommen wird und die aktuelle sowie kurzfristig erwartete Zahlungsfähigkeit des Unternehmens abbildet, die im Allgemeinen positiv mit der aktuellen Gewinnsituation korreliert ist. Diese Information liegt allerdings erst ab 1999 vor.

Da die im MUP erfassten Unternehmen aus Datenschutzgründen nicht mit Angaben aus den LAK- oder BA-Daten verknüpft werden können, kann kein $\mathrm{Maß}$ der individuellen Betroffenheit der Unternehmen vom Mindestlohn gebildet werden. Stattdessen werden Durchschnittswerte für die Betriebe des Dachdeckerhandwerks in einem regionalen Absatzgebiet und einer Beschäftigtengrößenklasse ermittelt und den Unternehmen im MUP zugespielt. Als regionale Absatzgebiete werden Landkreise bzw. kreisfreie Städte herangezogen. Als Indikatoren für die Betroffenheit vom Mindestlohn dienen zum einen der Anteil der gewerblichen Beschäftigten, die von der Einführung bzw. Erhöhung des Mindestlohns betroffen sind (Betroffenheitsquote), und zum anderen die betriebliche Lohnlücke. Durch den Einsatz von Paneldaten wird versucht, die unvermeidbare Endogenität zwischen der Mindestlohnvariable und anderen erklärenden Variablen im Bereich der Produktions- und Absatzbedingungen (da z. B. mindestlohnbedingte höhere Arbeitskosten zu Änderungen in den Produktionstechnologien oder der Nachfrage führen können) zu reduzieren.

Für die Untersuchung von Mindestlohneffekten ist es entscheidend, zwischen zwei Gruppen von Handwerksunternehmen zu unterscheiden, nämlich Unternehmen mit abhängig gewerblich Beschäftigten, die grundsätzlich vom Mindestlohn direkt betroffen sein können, und Unternehmen ohne solche Beschäftigte, die vom Mindestlohn nicht direkt betroffen sind. Da im MUP nur Informationen zur Anzahl der Mitarbeiter (inkl. Inhaber), aber nicht zum Status der Mitarbeiter vorliegen, werden diese Gruppen anhand der Anzahl der Mitarbeiter unterschieden. Mehr-PersonenUnternehmen (MPU) sind Unternehmen mit zwei oder mehr Mitarbeitern, wobei angenommen wird, dass diese zumindest einen abhängig gewerblich beschäftigten Mitarbeiter aufweisen. Ein-Personen-Unternehmen (EPU) sind definiert als Unternehmen, in dem nur der Inhaber tätig ist. Für MPU und EPU sind grundsätzlich unterschiedliche Mindestlohneffekte zu erwarten. Da EPU nicht vom Mindestlohn betroffen sind, gilt für sie der mindestlohnbedingte Arbeitskostenanstieg nicht, vielmehr könnten EPU versuchen, durch eine Niedrigpreisstrategie - sei es als Zuarbeiter von größeren
Betrieben, sei es im Verbund von mehreren EPU - Marktanteile auf Kosten der vom Mindestlohn betroffenen Unternehmen zu gewinnen. Auch können EPU eine Ausweichstrategie vom Mindestlohn darstellen. Der Anteil der EPU im Dachdeckerhandwerk auf Basis des MUP stieg von $7 \%$ im Jahr 1995 auf 22 \% im Jahr 2010 an. Für die Kontrollbranche des Installationshandwerks zeigt sich eine sehr ähnliche Entwicklung auf höherem Niveau (1995: $15 \%$, 2010: $32 \%$ ). Zusätzlich zur Differenzierung nach MPU und EPU ist eine Differenzierung nach Ost- und Westdeutschland zentral, da sich das Ausmaß des Mindestlohns in den beiden Regionen erheblich unterscheidet.

Die Schätzergebnisse zu den Mindestlohneffekten auf Profitabilität, Marktaustritte, Markteintritte und Unternehmensbestand sind in Tab. 5 dargestellt. Für Westdeutschland zeigen sich durchweg keine statistisch signifikanten Effekte der beiden betrachteten Mindestlohnvariablen (Betroffenheitsquote, betriebliche Lohnlücke). Dies ist angesichts der geringen Betroffenheit vom Mindestlohn, den sehr niedrigen Kostensteigerungen aufgrund des Mindestlohns und der großen Bedeutung anderer Einflussfaktoren auf den Wettbewerb im Dachdeckerhandwerk plausibel.

Für Ostdeutschland zeigen sich positive Profitabilitätseffekte für MPU, denen negative Effekte auf die Markteintritte von MPU und - zumindest für das Mindestlohnmaß Betroffenheitsquote ${ }^{12}$ - negative Effekte auf den Unternehmensbestand von MPU gegenüberstehen. Die Anzahl der Marktzutritte durch EPU und der Bestand an EPU wurden durch den Mindestlohn positiv beeinflusst. Diese Ergebnisse deuten darauf hin, dass der Mindestlohn in Ostdeutschland zu einer Verschiebung der Anbieterstruktur im Dachdeckerhandwerk zugunsten von EPU und zu Lasten von MPU geführt hat. Die beiden Unternehmenstypen scheinen jedoch nicht in einem direkten Preiswettbewerb zu stehen, da sich für die im Markt verbliebenen ostdeutschen MPU die Gewinnsituation relativ verbessert hat. ${ }^{13}$ Dies dürfte mit einem geringeren Wettbewerbsdruck zusammenhängen, da die mindestlohnbedingten geringeren Marktzutritte bei strukturell unveränderten Marktaustritten zu einer Verringerung des Unternehmensbestands (im Vergleich zur hypothetischen Situation ohne Mindestlohn) führten. Dabei ist zu

\footnotetext{
${ }^{12}$ Die Betroffenheitsquote bildet tendenziell langfristig und akkumulativ wirkende Mindestlohneffekte ab, da sie eng mit dem Anteil der Lohnkosten korreliert ist, die aufgrund des Mindestlohns höher liegen als ohne Mindestlohnregelung. Bei einem steigenden Anteil der von Mindestlohnerhöhungen betroffenen Mitarbeiter steigt diese Kostenbelastung von Jahr zu Jahr. Die betriebliche Lohnlücke gibt demgegenüber die kurzfristige Kostenerhöhung aufgrund der Erhöhung des Mindestlohns im Jahr t im Vorjahresvergleich an.

${ }^{13}$ Dies bedeutet nicht, dass sich die Gewinnsituation verbessert hat, das Gegenteil ist der Fall. Vielmehr haben sich die Gewinne der ostdeutschen MPU im Dachdeckerhandwerk aufgrund der Mindestlohneinführung weniger stark verschlechtert als dies ohne Mindestlohneinführung zu erwarten gewesen wäre.
} 
Tab. 5 Einfluss des Mindestlohns auf Profitabilität, Marktaustritte, Markteintritte und Unternehmensbestand im Dachdeckerhandwerk in Deutschland: Schätzergebnisse von Panelmodellen

\begin{tabular}{|c|c|c|c|c|c|}
\hline & & \multicolumn{2}{|c|}{ Betroffenheitsquote } & \multicolumn{2}{|c|}{ Betriebliche Lohnlücke } \\
\hline & & Koeffizient & $z$-Wert & Koeffizient & $z$-Wert \\
\hline \multicolumn{6}{|l|}{ Profitabilität ${ }^{\mathrm{a}}$} \\
\hline \multirow[t]{2}{*}{ Westdeutschland } & MPU & $-0,007$ & $-0,08$ & $-0,001$ & $-0,11$ \\
\hline & EPU & $-0,046$ & $-0,26$ & $-0,000$ & $-0,01$ \\
\hline \multirow[t]{2}{*}{ Ostdeutschland } & MPU & $0,232^{* *}$ & 4,67 & $0,026^{* *}$ & 2,75 \\
\hline & EPU & 0,127 & 1,15 & 0,015 & 0,80 \\
\hline \multicolumn{6}{|l|}{ Marktaustritt ${ }^{\mathrm{b}}$} \\
\hline \multirow[t]{2}{*}{ Westdeutschland } & MPU & 0,261 & 1,09 & 0,035 & 1,84 \\
\hline & EPU & $-0,054$ & $-0,50$ & $-0,039$ & $-1,31$ \\
\hline \multirow[t]{2}{*}{ Ostdeutschland } & MPU & 0,121 & 0,25 & $-0,028$ & $-0,60$ \\
\hline & EPU & 0,144 & 0,72 & 0,031 & 0,60 \\
\hline \multicolumn{6}{|l|}{ Gründungsrate $^{\mathrm{c}}$} \\
\hline \multirow[t]{2}{*}{ Westdeutschland } & MPU & $-0,026$ & $-0,98$ & 0,002 & 0,81 \\
\hline & EPU & 0,032 & 1,43 & 0,002 & 0,96 \\
\hline \multirow[t]{2}{*}{ Ostdeutschland } & MPU & $-0,041^{* *}$ & $-5,71$ & $-0,006^{* *}$ & $-2,63$ \\
\hline & EPU & $0,022^{* *}$ & 3,90 & $0,005^{*}$ & 1,97 \\
\hline \multicolumn{6}{|c|}{ Unternehmensbestand $^{\mathrm{d}}$} \\
\hline \multirow[t]{2}{*}{ Westdeutschland } & MPU & 0,015 & 0,65 & 0,000 & 0,13 \\
\hline & EPU & 0,085 & 1,34 & 0,013 & 1,52 \\
\hline \multirow[t]{2}{*}{ Ostdeutschland } & MPU & $-0,030^{* *}$ & $-2,78$ & $-0,002$ & $-0,43$ \\
\hline & EPU & $0,188^{* *}$ & 4,54 & $0,034^{*}$ & 2,42 \\
\hline
\end{tabular}

Alle Modelle enthalten Kontrollvariablen zu Unternehmensstrukturen, Angebots-, Wettbewerbs- und Nachfragebedingungen sowie Indikatorvariablen für Perioden, Raumordnungsregionen und Branche (Dachdecker)

${ }^{a}$ Ordinalvariable, gemessen in neun Bonitätsstufen; geordnete Probitmodelle, Stützperiode 1999-2010; 535.898 Beobachtungen zu 77.525 unterschiedlichen Unternehmen (15.009 Dachdecker- und 62.516 Installationsunternehmen)

${ }^{b}$ Binäre Variable (1 falls Marktaustritt im Beobachtungsjahr, sonst 0); komplementäre Log-Log-Modelle, Stützperiode 1996-2010; 780.457 höher (92.478 unterschiedliche Unternehmen, darunter 18.110 Dachdecker- und 74.368 Installationsunternehmen)

${ }^{\mathrm{c}}$ Anzahl der Gründungen je Unternehmensbestand im regionalen Absatzmarkt; Tobit-Regressionen, Stützperiode 1996-2010; 11.446 Beobachtungen auf Basis von 413 Kreisen

dAnzahl der wirtschaftsaktiven Unternehmen (log) im regionalen Absatzmarkt; OLS-Regressionen, Stützperiode 1996-2010; 11.551 Beobachtungen auf Basis von 413 Kreisen

MPU: Mehr-Personen-Unternehmen; EPU: Ein-Personen-Unternehmen

** $\left(^{*}\right)$ : Die geschätzten Koeffizienten sind mit einer Fehlerwahrscheinlichkeit von $1 \%(5 \%)$ statistisch signifikant

beachten, dass der Wettbewerbsdruck bei Einführung des Mindestlohns sehr hoch war, da ab 1995 die Bautätigkeit in Ostdeutschland stark nachließ und es zu Überkapazitäten kam.

Eine Voraussetzung für diese Entwicklung ist, dass die im Markt verbliebenen MPU in Ostdeutschland zumindest einen Teil der mindestlohnbedingten Kostenerhöhung entweder über höhere Preise an die Nachfrager weitergeben oder über Produktivitätserhöhungen intern abfedern konnten. Unsere Analysen zu den Mindestlohneffekten auf die Güterpreise liefern einen schwachen Beleg für eine partiel- le Weitergabe höherer Kosten über höhere Preise. ${ }^{14}$ Nur für

\footnotetext{
${ }^{14}$ Abhängige Variable ist die Entwicklung des Baupreisindexes für Dachdeckungsarbeiten sowie für Installationsarbeiten (als Kontrollbranche) für Deutschland insgesamt (Quartalsdaten, Quelle: Statistisches Bundesamt). Es wurden über vier Preisindexreihen (Schönheitsreparaturen in Mehrfamiliengebäuden, Hochbauarbeiten für Wohngebäude, Hochbauarbeiten für Bürogebäude und Hochbauarbeiten für gewerbliche Betriebsgebäude) gepoolte Modelle für zwei Varianten (Veränderungsrate der Preise und absolute Höhe der Preisindizes) separat für die beiden Mindestlohnindikatoren (Betroffenheitsquote und Lohnlücke, jeweils Quartalsmittelwerte für alle Unternehmen in Deutschland, wobei für das 1. Quartal der März- und für das 4. Quartal der
} 
eine der vier Modellvarianten konnte ein positiver und statistisch signifikanter Einfluss (nämlich der Betroffenheitsquote auf den Preisindex) gefunden werden, die anderen Modellschätzungen erbrachten insignifikante Ergebnisse.

Die Ergebnisse zu den Wettbewerbswirkungen des Mindestlohns im Dachdeckerhandwerk sollten vor dem Hintergrund der nicht optimalen Datenlage mit Vorsicht interpretiert werden. So ist die regionale Marktabgrenzung, die für die Analysen zu Markteintritten und Unternehmensbestand zentral ist, problematisch, da sich der regionale Aktionsradius je nach Unternehmen unterscheiden kann. Auch können die empirischen Modelle die relevanten Einflussfaktoren des Wettbewerbs im Markt für Dachdeckerleistungen sowie in der Kontrollbranche des Installationsgewerbes nur unvollständig abbilden. Dadurch besteht die Gefahr, dass nicht beobachtbare Einflussfaktoren mit dem beobachtbaren Mindestlohnmaß korrelieren und deren Effekte auf das Mindestlohnmaß abgeschoben werden. Dies gilt insbesondere angesichts des Umstandes, dass der Mindestlohneffekt über die Zeit tendenziell ansteigt (da die Betroffenheit zunimmt und die Erhöhung des Mindestlohns meist über der der übrigen Tariflöhne lag) und sich gleichzeitig auch die Marktsituation im Dachdeckerhandwerk seit Mitte der 2000er Jahre erheblich verbessert hat, wodurch die Zahl der Marktaustritte zurückging und Gewinne und Gründungszahlen stabilisiert wurden. Außerdem war es nicht möglich, die unternehmensspezifische Betroffenheit durch den Mindestlohn zu berücksichtigen.

\section{Wirtschaftspolitische Schlussfolgerungen}

Der 1997 eingeführte und seit 2003 bundeseinheitlich geregelte Mindestlohn im Dachdeckerhandwerk führte vor allem in Ostdeutschland zu einer auch im internationalen Vergleich sehr hohen Eingriffsintensität des Mindestlohns. 2008 erhielten in Westdeutschland etwa $10 \%$ der gewerblich abhängigen Beschäftigten im Dachdeckerhandwerk einen Lohn unterhalb des nächstgeltenden Mindestlohns, in Ostdeutschland machte diese Gruppe mehr als $50 \%$ der Beschäftigten aus. Während daher der durchschnittliche vom Mindestlohn betroffene Beschäftigte in Westdeutschland eher ein atypisch Beschäftigter ist, der nicht zu der vollzeitbeschäftigten Stammbelegschaft gehört, erfasst der Mindestlohn in Ostdeutschland auch den durchschnittlichen Facharbeiter.

\footnotetext{
Oktoberwert genommen wurde, um mögliche Saisoneinflüsse zu minimieren) sowie weiteren Kontrollvariablen (Vorleistungspreise, mindestlohnunabhängige Arbeitskosten, Auftragseingangsvolumen, $\mathrm{Zu}-$ bau an Photovoltaikanlagen auf Dächern) geschätzt (Stützperiode: 2 . Quartal 1996 bis 2. Quartal 2009). Eine Differenzierung nach Ost- und Westdeutschland ist nicht möglich.
}

Die mit dem Mindestlohn einhergehende effektive Kostenbelastung fiel dennoch gering aus. Zum einen führt der Mindestlohn selbst bei vollständiger Einhaltung zu Gesamtkostenerhöhungen von lediglich $0,3-0,8 \%$ in Ostdeutschland und $0,2-0,5 \%$ in Westdeutschland. Zum anderen wurde der Mindestlohn vielfach nicht vollständig eingehalten.

Die Wirkungsanalysen zu den Mindestlohnwirkungen auf die Wettbewerbssituation im Markt für Dachdeckerleistungen erbrachten keine eindeutigen Ergebnisse, was unter anderem auf die eingeschränkte Datenbasis zurückzuführen ist. Auf der Ebene der Preise liegen gewisse Hinweise vor, dass die mindestlohnbedingten Kostensteigerungen zumindest teilweise und eher mittelfristig über höhere Preise an die Kunden weitergegeben wurden. Für Westdeutschland zeichnen sich keine mindestlohnbedingten Wirkungen auf Profitabilität, Eintritte, Austritte oder Unternehmensbestand ab. In Ostdeutschland scheint sich die Gewinnsituation für Mehr-Personen-Unternehmen aufgrund des Mindestlohns etwas verbessert zu haben, was auf niedrigere Marktzutritte und einen abnehmenden Unternehmensbestand zurückgeführt werden könnte. Außerdem zeigen die Wirkungsanalysen, dass der Mindestlohn in Ostdeutschland zu einer leichten Verschiebung der Unternehmensstruktur in Richtung Ein-Personen-Unternehmen (zulasten von MehrPersonen-Unternehmen) beigetragen hat.

Die Wirkungsanalysen zum Arbeitnehmerschutz belegen, dass der Mindestlohn in Ostdeutschland zu deutlichen Stundenlohnzuwächsen für die unteren Dezile der Lohnverteilung geführt hat. Auch in Westdeutschland zeigen sich für die vom Mindestlohn betroffenen unteren zwei Dezile überdurchschnittliche Lohnzuwächse. Diese Lohnzuwächse übersetzten sich jedoch nicht Eins zu Eins in Einkommenszuwächse, da die Arbeitsstunden der vom Mindestlohn betroffenen Beschäftigten im gleichen Zeitraum tendenziell sanken. Dennoch stiegen die Tageseinkommen der vom Mindestlohn betroffenen Beschäftigten im Vergleich zur Installationsbranche vor allem in Ostdeutschland an.

In Ostdeutschland steht dem Einkommenszuwachs der vom Mindestlohn betroffenen Beschäftigten ein Einkommensverlust seitens der oberen Lohndezile gegenüber. Diese Entwicklung ist das Resultat weitgehend stagnierender Nominallöhne und einer in den letzten Jahren insgesamt reduzierten Arbeitszeit. Diese sinkenden Arbeitskosten für die besser verdienenden Beschäftigten der Branche halfen somit, die mindestlohnbedingten Kostensteigerungen am unteren Rand zu kompensieren. Eine Lohndifferenzierung findet in Ostdeutschland somit kaum mehr statt, eine Entwicklung die sowohl von Arbeitnehmer- als auch Arbeitgebervertretern als kritisch eingeschätzt wird, da die Attraktivität eine Dachdeckerlehre zu beginnen schwindet, wenn Fachkräfte nicht ausreichend mehr verdienen als Ungelernte. Problematisch sehen vor allem die Arbeitnehmervertreter die Tendenz, dass der Mindestlohn zu einer Orientierungsmarke und damit in Ostdeutschland weitgehend zum Normlohn 
geworden ist. Die Verbandsvertreter sind sich jedoch sicher, dass der aktuell zunehmende, auch abwanderungsbedingte Fachkräftemangel diese Praxis in der Zukunft erschwert und ein Umdenken in den Betrieben einsetzen könnte.

Die Analysen weiterer Indikatoren des Arbeitnehmerschutzes auf Basis der eigenen Unternehmensbefragung konnten keine mindestlohnbedingten Veränderungen in der Vergütung von Überstunden, der Bedeutung prekär Beschäftigter in der Randbelegschaft oder dem Krankenstand im Unternehmen finden. Dies bedeutet entweder, dass es hier keine Auswirkungen gegeben hat oder dass die Datenlage nicht ausreichte, Wirkungen des Mindestlohns nachzuweisen.

Die Gesamtbeschäftigung der Branche hat sich mindestlohnbedingt vermutlich nicht verändert. Analysen zu den Wirkungen des Mindestlohns auf die Gesamtbeschäftigung auf der Ebene regionaler Märkte weisen zumeist keine statistisch signifikanten Effekte aus. Für die vom Mindestlohn betroffenen Beschäftigten und Betriebe lassen sich im Vergleich zu den nicht direkt betroffenen Beschäftigten und Betrieben sowohl in West- als auch Ostdeutschland jedoch negative Beschäftigungsfolgen nachweisen. Der nicht vorhandene Effekt auf der Ebene des (regionalen) Marktes deutet jedoch darauf hin, dass diese Wirkungen sich nicht in Nettoverlusten in der Branche niederschlugen, da nicht betroffene Fachkräfte und/oder andere Betriebe der Branche indirekt profitieren und Beschäftigungsverluste in Teilen der Branche ausgleichen. $\mathrm{Ob}$ die zu beobachtende Fachkräfteintensivierung jedoch vollständig dem Mindestlohn zuzurechnen ist, kann nicht abschließend geklärt werden. Für eine Wirkung des Mindestlohns spricht, dass bei einer in den letzten Jahren extrem gesunkenen Lohnspreizung die Beschäftigung von Fachkräften im Vergleich zu ungelernten Arbeitskräften extrem an Attraktivität gewonnen hat. Langfristig kann dies jedoch zu Lasten der Attraktivität einer Dachdeckerlehre gehen und damit zu einem Fachkräftemangel führen, wenn die Ausbildungsrendite sinkt. Die eigene Unternehmensbefragung liefert erste Evidenzen für einen sich abzeichnenden Fachkräftemangel im Dachdeckerhandwerk. Demnach kommen derzeit auf 100 Beschäftigte im Durchschnitt 10,6 offene Stellen, die kurzfristig nicht besetzt werden können.

Es lassen sich somit weder starke Wettbewerbswirkungen noch Nettobeschäftigungseffekte nachweisen. Dies kann einerseits auf die jeweiligen Schwächen der Analyse zurückzuführen sein, ist andererseits aber auch ökonomisch durchaus plausibel, da effektive Arbeitskostenerhöhungen, die nicht durch Preisweitergaben kompensiert werden konnten, vermutlich eher gering ausfielen. Hinzu kommt, dass der technische Fortschritt im Dachdeckerhandwerk die Ausschöpfung von Produktivitätsreserven - laut Verbandsvertretern im Osten in größerem Maße vorhanden als im Westen - vermutlich begünstigte und auf diesem
Wege ebenfalls zur Kompensation steigender Arbeitskosten beitrug. Immerhin ein Drittel der befragten Unternehmen gibt zudem an, dass der Mindestlohn zu einer erhöhten Motivation der Beschäftigten beigetragen habe, so dass auch auf diesem Wege teilweise Produktivitätsreserven realisiert werden konnten. Darüber hinaus half in Ostdeutschland eine Lohnzurückhaltung für Fachkräfte einen Teil der zusätzlichen Arbeitskosten zu kompensieren.

Die teilweise moderaten Auswirkungen des Mindestlohns im Dachdeckergewerbe sind somit auch vor dem Hintergrund der branchenspezifischen Kompensationsmöglichkeiten mindestlohnbedingter Arbeitskosten zu sehen. Die Ergebnisse können somit keinesfalls als wahrscheinliche Wirkungen eines flächendeckenden gesetzlichen Mindestlohns interpretiert werden, sondern spiegeln die Besonderheiten der Branche und das aktuelle Marktumfeld wider. Mit veränderten Rahmenbedingungen können sich jedoch auch die Wirkungen des Mindestlohns verändern.

So reduziert die fehlende Lohndifferenzierung seit einigen Jahren die Attraktivität einer Ausbildung zum Dachdeckergesellen und verstärkt somit möglicherweise den aktuell stark zunehmenden Fachkräftemangel in der Branche. Während die bisherigen Beschäftigungswirkungen somit in eine Phase des Arbeitskräfteüberschusses nach dem Ende des wiedervereinigungsbedingten Baubooms fielen, können Betriebe in der aktuellen Situation Fachkräfte vermehrt nur durch verbesserte Arbeitsbedingungen halten. In einem solchen Marktumfeld könnten die Auswirkungen des Mindestlohns auf die Nettobeschäftigung jedoch zunehmen. Zudem wäre auch im Falle einer vollständigen Einhaltung des Mindestlohns möglicherweise mit stärkeren Auswirkungen auf die Nettobeschäftigung zu rechnen.

Trotz der ein oder anderen nicht intendierten Wirkung des Mindestlohns im Dachdeckerhandwerk steht die Branche einem allgemeinverbindlichen Mindestlohn weitgehend positiv gegenüber. Sowohl Arbeitnehmer- als auch Arbeitgebervertreter sind der Meinung, dass der Mindestlohn eine wichtige untere Haltelinie für den Preiswettbewerb darstellt, der somit Wettbewerbsverzerrungen zu Lasten der Arbeitnehmer/innen reduziere. Die Durchführung von Kontrollen stellt dafür jedoch eine Grundvoraussetzung dar. Aus der Sicht der Verbände reichen die Bemühungen der Kontrollinstanzen und die Sanktionsmöglichkeiten bislang nicht aus.

\section{Zusammenfassung}

In der Dachdeckerbranche wurde 1997 ein allgemeinverbindlicher Mindestlohn eingeführt, der seitdem mehrfach erneuert und angehoben wurde und im Jahr 2011 10,80€ betrug. Als erste Branche wies das Dachdeckerhandwerk zudem ab 2003 einen bundeseinheitlichen Mindestlohn auf. Diese besondere Situation führt zu einer in Ostdeutschland 
sehr hohen Betroffenheit vom Mindestlohn; etwa $50 \%$ der ostdeutschen gewerblichen Beschäftigten der Branche erzielen einen Verdienst zum Mindestlohnniveau. Die Höhe des Mindestlohns gemessen am Medianlohn der Branche ist damit so hoch wie in keiner anderen Branche in Deutschland.

Auf der Basis von Differenz-von-Differenzen-Schätzungen sowohl im Vergleich zu einer nicht von einem Mindestlohn betroffenen Baunebenbranche als auch auf Basis eines Vergleichs von unterschiedlich stark durch den Mindestlohn betroffenen Beschäftigten des Dachdeckerhandwerks werden in diesem Beitrag die kausalen Wirkungen im Hinblick auf Beschäftigung, Arbeitnehmerschutz und Wettbewerb untersucht. Für diese Analysen steht mit den administrativen Arbeitnehmer-Arbeitgeberdaten der Bundesagentur für Arbeit, dem Mannheimer Unternehmenspanel des ZEW sowie der Vollerhebung der Beschäftigten des Dachdeckerhandwerks durch die Lohnausgleichskasse des Dachdeckerhandwerks eine sehr gute Datengrundlage zur Verfügung. Insbesondere kann die Betroffenheit vom Mindestlohn wesentlich genauer festgestellt und für kausale Wirkungsanalysen genutzt werden, als dies in Studien für andere Branchen möglich war.

Die Ergebnisse der Evaluation zeigen, dass es vor allem in Ostdeutschland mindestlohnbedingt zu deutlichen Stundenlohnzuwächsen für die unteren Dezile der Lohnverteilung kam. Diese Lohnzuwächse übersetzten sich nur teilweise in Einkommenszuwächse, da die Arbeitsstunden der vom Mindestlohn betroffenen Beschäftigten im gleichen Zeitraum tendenziell sanken. Zudem stehen den Einkommenszuwächsen der vom Mindestlohn betroffenen Beschäftigten Einkommensverluste seitens der oberen Lohndezile gegenüber. Dies trug möglicherweise dazu bei, dass sich zwar die Beschäftigungschancen der mindestlohnbedingt verteuerten Arbeitskräfte verschlechtert haben, die Gesamtbeschäftigung jedoch vermutlich unverändert blieb. Eindeutige Wettbewerbswirkungen ließen sich nicht nachweisen, wenngleich sich für Ostdeutschland eine gewisse Verschiebung der Unternehmensstruktur in Richtung Ein-PersonenUnternehmen zeigt. Zudem gibt es Hinweise dafür, dass die mindestlohnbedingten Kostensteigerungen zumindest teilweise über höhere Preise an die Kunden weitergegeben wurden.

Insgesamt können die Ergebnisse nicht als wahrscheinliche Wirkungen eines flächendeckenden gesetzlichen Mindestlohns interpretiert werden, sondern spiegeln die Besonderheiten der Branche und das aktuelle Marktumfeld wider. Mit veränderten Rahmenbedingungen können sich auch die hier gezeigten Wirkungen des Mindestlohns verändern.

\section{Executive summary}

Generally binding minimum wages were introduced in the roofing sector in 1997 and have been renewed and raised several times since then; in 2011, the roofer minimum wage amounted to $11.80 €$. Moreover, the roofing sector was the first to introduce national minimum wages in 2003. This specific situation lead to a hard bite of the minimum wage in Eastern Germany; about $50 \%$ of all East German blue-collar workers earn a wage at minimum wage level. The minimum wage level as compared to the median wage of the sector is thus higher than in all other sectors in Germany.

Based on difference-in-differences estimations in comparison to an ancillary construction sector that is not affected by minimum wages as well as based on a comparison of workers in the roofing sector who have been affected by minimum wage introduction to different degrees, this paper investigates the causal effects with regard to employment, worker protection and competition. The administrative employee-employer data of the Federal Employment Agency, the Mannheim business panel of the ZEW, as well as the full-scale survey of all employees in the roofing sector conducted by the income supplement fund of the roofing trade provide a well-documented database for these analyses. In contrast to previous studies for other sectors, minimum wage bite can be detected more precisely and can be used for causal effect analyses.

The results show that, particularly in East Germany, minimum wages cause considerable increases in hourly wages for lower deciles of the wage distribution. These wage increases have only partially compiled into earnings increases because the working hours of those affected by minimum wages have decreased by tendency at the same time. Furthermore, wage increases caused by minimum wage introduction are opposed to wage decreases in the upper deciles. This might have contributed to the fact that employment opportunities for workers, who are now more expensive due to minimum wages, have decreased, while the overall employment has presumably not changed. Distinct competitive impacts could not be detected, even though there is a slight tendency toward single-person companies in the East German corporate structure. Moreover, there is evidence that cost increases due to minimum wages were, at least in part, transferred to the customers via increased prices.

To conclude, the results cannot be interpreted as probable effects of national statutory minimum wages, but they rather present the distinctiveness of the sector and the current market environment. Changing the framework conditions could also change the minimum wage effects shown above.

Danksagung Wir danken Jürgen Egeln, Sandra Gottschalk, Kornelius Kraft, Nina Leheyda, Helmut Schröder, Holger Schütz und Stephan Dlugosz für hilfreiche Diskussionen und Unterstützung bei den Analysen sowie Frau Nitzling für die Unterstützung im Umgang mit den Daten der Lohnausgleichskasse des Dachdeckerhandwerks. 


\section{Anhang A: Datenbasis}

\section{A.1 Mannheimer Unternehmenspanel (MUP-Daten)}

Das Mannheimer Unternehmens Panel (MUP) ist eine vom ZEW in Zusammenarbeit mit Creditreform, der größten Kreditauskunftei in Deutschland, aufgebaute Paneldatenbank, die nahezu alle wirtschaftsaktiven Unternehmen zum Zeitpunkt der Wirkungsanalysen (Frühjahr 2011) 3,06 Mio. aktive von insgesamt 6,8 Mio. Unternehmen (einschließlich in früheren Jahren geschlossene Unternehmen) enthält. Damit bildet das MUP die Grundgesamtheit der Unternehmen in Deutschland - inklusive Kleinstunternehmen und selbstständige Freiberufler - ab. Die statistische Einheit des MUP ist das rechtlich selbstständige Unternehmen. Im Bereich der hier interessierenden Branchen Dachdecker und Installateure zeigt ein Vergleich mit der Umsatzsteuerstatistik, dass der Anteil der im MUP erfassten Dachdeckerund Installateurunternehmen im Mittel der betrachteten Periode $99,9 \%$ bzw. 116,6 \% der in der Umsatzsteuerstatistik erfassten Unternehmen ausmacht, d. h. das MUP erfasst bei Installateuren zusätzliche (i. d. R. Ein-Personen-) Unternehmen, die unterhalb der Meldegrenze der Umsatzsteuerstatistik (i. d. R. $17.500 €$ Jahresumsatz) bleiben. Diese „Übererfassung“ gilt für alle Beobachtungsjahre. Das Verhältnis zwischen den im MUP erfassten Unternehmen und den in der Umsatzsteuerstatistik erfassten Unternehmen ist zu Beginn und zu Ende der Beobachtungsperiode sowohl bei Dachdeckern als auch bei Installateuren jeweils etwas niedriger, was auf eine methodisch bedingte Untererfassung durch Creditreform zurückzuführen ist.

Das MUP wird halbjährlich aktualisiert und erlaubt u. a. die Verfolgung der Entwicklung von Unternehmen seit deren Gründung, einschließlich der Identifikation von verschiedenen Typen von Markt-austritten durch Unternehmensschließung. Mit Hilfe des MUP kann die Entwicklung der Gründungszahlen seit 1995, der Schließungszahlen seit 2000 und des Unternehmensbestands seit 2000 differenziert nach verschiedenen Strukturmerkmalen der gegründeten und geschlossenen Unternehmen und der Gründerpersonen untersucht werden. Für jedes Unternehmen liegen Branchenklassifikationen nach Wirtschaftszweigen sowie eine Kurzbeschreibung der Geschäftstätigkeit vor. Außerdem enthält das MUP u. a. jährliche Informationen (ab 1999) zum Umsatz, zur Anzahl der Mitarbeiter, aber auch zur Bonität von Unternehmen, eine Maßzahl, die sich als Indikator für die wirtschaftliche Situation der Unternehmen eignet.

\section{A.2 Daten der Lohnausgleichskasse der Dachdeckerhandwerks (LAK-Daten)}

Seit 1995 existiert im Dachdeckerhandwerk eine allgemeinverbindliche Teilnahme am Sozialkassenverfahren des
Dachdeckerhandwerks, welches u. a. der Abwicklung des 13. Monatsgehalts, der Altersversorgung, der Berufsbildung und Beschäftigungssicherung während der Wintermonate dient. Alle inländischen Betriebe und selbstständigen Betriebsabteilungen des Dachdeckerhandwerks sind demnach verpflichtet, ihre gewerblichen Beschäftigten mit der Angabe der tatsächlichen, entlohnten Arbeitszeit und der dazugehörigen Bruttolohnsumme monatlich zu melden. Diese Monatsmeldungen stehen für die Zeit von 1995 bis 2010 zur Verfügung. Für jeden Beschäftigten enthalten die Daten neben der monatlichen Arbeitszeit und dem Monatslohn Informationen zu Alter, Geschlecht, Gründe für temporäre Abwesenheiten (z. B. Krankheit, Wehrpflicht) sowie Beginn und Ende der Beschäftigung im Betrieb.

Da unter den gemeldeten gewerblichen Beschäftigten Azubis und Reinigungskräfte nicht identifiziert werden können, ist eine exakte Abgrenzung der den Mindestlohnregelungen unterliegenden Beschäftigten somit schwierig. Diese beiden Gruppen können durch die Nichtberücksichtigung minderjähriger Beschäftigter sowie der Eingrenzung auf männliche Beschäftigte jedoch vermutlich weitgehend ausgeschlossen werden. Die wenigen weiblichen Dachdeckerinnen, die unter die Regelung fallen, werden in den LAK Daten daher jedoch nicht berücksichtigt. Insgesamt umfassen die Juni-Daten 1,1 Mio. Personenbeobachtungen in 23.000 Dachdeckerbetrieben, die zwischen 1995 und 2010 mindestens einen gewerblich Beschäftigten hatten. Die in den Daten vorhandenen Betriebs- und Personenidentifikationsnummern erlauben es zudem, die Beschäftigtenstruktur eines Betriebes zu beobachten und zudem den Verbleib eines Individuums nicht nur im Betrieb, sondern auch in der Branche zu verfolgen.

Bei der Interpretation der aus den Daten berechneten Stundenlöhne ist zu beachten, dass diese aufgrund von Beschäftigungssicherungsmaßnahmen zwischen Oktober und April in diesen Monaten keineswegs dem tatsächlichen Stundenlohn entsprechen müssen. Aus diesem Grund werden primär die Informationen für den Monat Juni ausgewertet. Der Stundenlohn in diesem Monat kann jedoch durch Überstundenzuschläge und Sonderzahlungen geringfügig überschätzt sein.

\section{A.3 Daten der Bundesagentur für Arbeit (BA-Daten)}

Die Daten setzen sich aus der Beschäftigten-Historik (BeH) und dem Betriebs-Historik-Panel (BHP) zusammen. Beim BHP handelt es sich um ein Panel aus Betriebsinformationen zum Stichtag 30.06., die durch Aggregation der zu dem Betrieb gehörenden Beschäftigtenmeldungen entstehen (vgl. Hethey-Maier und Seth 2010). Hierfür werden aus allen in den Jahren 1994 bis 2008 vorkommenden Dachdeckerbetrieben $75 \%$ und allen Installationsbetrieben aufgrund der höheren Fallzahl $30 \%$ als Stichprobe gezogen. Für jeden Betrieb sind Informationen zum Wirtschaftszweig, dem 
Bundesland sowie Angaben zur Gründung und Schließung des Betriebs vorhanden. Darüber hinaus stehen aggregierte Stichtagsinformationen zu der Anzahl und der Struktur der Beschäftigten des Betriebs zur Verfügung.

Aus der $\mathrm{BeH}$, den Beschäftigtenmeldungen der Betriebe an die Sozialversicherungsträger, werden zunächst alle sozialversicherungspflichtig Beschäftigten zugespielt, die im Zeitraum 1994 bis 2008 an mindestens einem Stichtag (30.06.) in einem der aus dem BHP gezogenen Betriebe beschäftigt waren. Damit umfasst die BeH alle Arbeiter/innen, Angestellten und Auszubildenden, die nicht von der Sozialversicherungspflicht befreit sind. Seit der Änderung des Meldeverfahrens zum 01.01.1999 werden auch geringfügig Beschäftigte erfasst. Unternehmen werden in den Daten daher erst sichtbar, wenn sie mindestens eine/n sozialversicherungspflichtige/n (oder ab 1999 geringfügig) Beschäftigte/n umfassen.

Insgesamt stehen nach Ziehung der Stichprobe im Geltungsbereich des Mindestlohns etwa 790.000 Dachdeckerbeobachtungen in 17.000 Dachdeckerbetrieben sowie 1,5 Mio. Beobachtungen der Installationsbranche in 35.000 Installationsbetrieben zur Verfügung. Für jeden Beschäftigten sind Schul- und Berufsausbildung, Alter, Geschlecht, die berufliche Stellung sowie dessen Beruf bekannt. Die tatsächlich den Mindestlohnregelungen unterliegenden gewerblichen Beschäftigten lassen sich auf Basis der Informationen in den BA-Daten somit nahezu exakt identifizieren. Darüber hinaus beinhalten die Daten das Bruttotagesentgelt, welches sich als Durchschnittsverdienst für die Dauer des Beschäftigungsverhältnisses innerhalb eines Jahres ergibt. Da jedoch nur zwischen Vollzeit- und Teilzeitbeschäftigten unterschieden werden kann, fehlen die exakten Stundenlöhne, die für die Wirkungsanalysen jedoch eine zentrale Information darstellen. Die Stundenlöhne werden daher unter Verwendung der LAK-Daten imputiert (siehe Anhang B).

\section{A.4 CATI-Telefonbefragung}

Aufgrund fehlender Zielgrößen in den administrativen Daten, z. B. im Bereich des Arbeitnehmerschutzes, wurde zusätzlich eine telefonische Unternehmensbefragung (CATI) durchgeführt. Für die standardisierte und mit einer Dauer von zehn Minuten relativ kurze Befragung wurden dazu von infas (Institut für angewandte Sozialwissenschaft, Bonn) 250 Unternehmen der Dachdeckerbranche und 250 Unternehmen der Installationsbranche aus dem MUP befragt. Um bei der Auswertung der Befragungsergebnisse Aussagen zu Unterschieden zwischen Ost- und Westdeutschland sowie zwischen unterschiedlich großen Unternehmen treffen zu können, wurde nach der Region - West- und Ostdeutschland - sowie nach drei Unternehmensgrößenklassen - 1 bis 5, 6 bis 40 , mehr als 40 Mitarbeiter - geschichtet. Die Unternehmen wurden zu Umfang und Struktur der betrieblichen Be- schäftigung, zu Entlohnungs- und Arbeitsbedingungen sowie ihrer Wettbewerbssituation befragt. Auch die Einschätzungen und Reaktionen auf den Mindestlohn wurden erhoben.

\section{Anhang B: Imputationsverfahren}

Die Bruttostundenlöhne in den BA-Daten wurden mithilfe der LAK-Daten rekonstruiert. Zu diesem Zweck wird der LAK-Datensatz in seiner Datenstruktur zunächst dem BeH-Datensatz angepasst, indem für die LAK-Daten aus den Monatsinformationen und den genauen Informationen zum Anfang- und Endzeitpunkt einer Beschäftigung eine jährliche Beschäftigungsmeldung generiert wird, die in ihrer Struktur mit dem BeH-Datensatz übereinstimmt. AnschlieBend wird der Stundenlohn in den LAK-Daten in Abhängigkeit von Merkmalen ${ }^{15}$ geschätzt, die auch in den BeH-Daten zur Verfügung stehen. Die geschätzten Koeffizienten können somit genutzt werden, um den fehlenden Stundenlohn in den BeH-Daten für jedes Individuum zu prognostizieren. Um einen möglichst hohen Erklärungsgehalt des Imputationsmodells sowie eine große Flexibilität in der Reststreuung zu gewährleisten, werden die Schätzungen für Westund Ostdeutschland, große (mehr als 10 Beschäftigte) und kleine Unternehmen (höchstens 10 Beschäftigte), die Jahre 1995 bis 2008 und die Quintile der Tagesentgeltverteilung jeweils getrennt durchgeführt. ${ }^{16}$ Dieses Vorgehen gewährleistet eine hohe Prognosequalität der Imputation, da insgesamt 88 Prozent der Gesamtvarianz des Stundenlohns im Beobachtungszeitraum mit den Schätzmodellen erklärt werden. Die Verteilung der imputierten mittleren Stundenlöhne in den BA-Daten entspricht daher weitgehend der Verteilung in den LAK-Daten (vgl. Aretz et al. 2011).

Zusätzlich zum prognostizierten mittleren Stundenlohn erhält jedes Individuum in den BA Daten eine prognostizier-

\footnotetext{
${ }^{15}$ Die Kovariate sind zum einen individuelle Charakteristika wie der Tageslohn und Jahreslohn, Geschlecht, Alter, Bundesland sowie Informationen zur Länge der Betriebs-, Branchen- und Stichprobenzugehörigkeit. Auf der Betriebsebene stehen Informationen wie die Unternehmensgröße, der Anteil der schlechter verdienenden Beschäftigten im Betrieb sowie der Anteil der temporären Saisonarbeitskräfte im Betrieb zur Verfügung. Darüber hinaus wird ein Interaktionsterm in der Schätzung berücksichtigt, der sich aus dem Start und der Länge der Beschäftigungsperiode in einem Jahr zusammensetzt. Dies kontrolliert für saisonale Schwankungen der Arbeitszeit und der Lohnzuschläge, da ein und derselbe Tageslohn für eine dreimonatige Beschäftigung in den Sommer- oder Wintermonaten mit einem unterschiedlichen Stundenlohn einhergehen dürfte.

16 Ähnliche Imputationstechniken werden in der Literatur häufig verwendet, um fehlende Beobachtungen innerhalb eines Datensatzes zu füllen, siehe z. B. Little und Rubin (2002). Dass zwei Datensätze verwendet werden, deren Personen vielfach identisch sind, ist zwar unüblich, verbessert jedoch die Qualität der Imputation gegenüber Standardtechniken.
} 
te Varianz des Stundenlohns, welche die statistische Unsicherheit in den geschätzten Koeffizienten sowie die als normalverteilt angenommene Residualstreuung widerspiegelt. Auf Basis dieser imputierten Informationen lässt sich für jedes Individuum die Wahrscheinlichkeit bestimmen, einen Stundenlohn unterhalb des (nächstgeltenden) Mindestlohns zu erhalten und somit von einem bindenden Mindestlohn betroffen zu sein. Anders als in den LAK-Daten verfügen wir somit nicht über die exakte Betroffenheit vom Mindestlohn auf der individuellen Ebene, sondern nur über eine entsprechende Wahrscheinlichkeit. Zwar folgt das Muster der mittleren Betroffenheitswahrscheinlichkeit weitgehend dem Muster des auf Basis der LAK-Daten bestimmten Anteils von Beschäftigten mit einem bindenden Mindestlohn; zumindest für Ostdeutschland ergibt sich in den letzten Jahren aufgrund der extremen Lohnstauchung in Ostdeutschland jedoch eine zunehmende Unterschätzung der Betroffenheit, siehe Aretz et al. (2011). Trotz dieser Abweichungen sollte die imputierte Betroffenheitswahrscheinlichkeit stark mit der tatsächlichen individuellen Lohnlücke des Beschäftigten zusammenhängen. Verschiedene Robustheitsanalysen in Aretz et al. (2011) bestätigen zudem, dass Analysen auf Basis beider Datensätze zu sehr vergleichbaren Ergebnissen kommen.

\section{Literatur}

Ai, C., Norton, E.: Interaction terms in logit and probit models. Econ. Lett. 80(1), 123-129 (2003)

Aretz, B., Arntz, M., Gottschalk, S., Gregory, T., Niefert, M., Rammer, C., Schröder, H., Schütz, H.: ZEW-Studie zu den Wirkungen eines Mindestlohns in der Dachdeckerwirtschaft. Research report for the Federal Ministry of Labour and Social Affairs, ZEW Mannheim (2011)

Cameron, A., Trivedi, P.: Microeconometrics: Methods and Applications. Cambridge University Press, Cambridge (2005)

Card, D.: Using regional variation in wages to measure the effects of the federal minimum wage. Ind. Labor Relat. Rev. 46(1), 22-37 (1992)

Card, D., Krueger, A.: Minimum wages and employment: a case study of the fast-food industry in New Jersey and Pennsylvania. Am. Econ. Rev. 84(4), 772-793 (1994)

Card, D., Krueger, A.: Myth and Measurement: the New Economics of the Minimum Wage. Princeton University Press, Princeton (1995)

DiNardo, J., Fortin, N., Lemieux, T.: Labor market institutions and the distribution of wages, 1973-1992: a semiparametric approach. Econometrica 64(5), 1001-1044 (1996)

Dolton, P., Rosazza-Bondibene, C.: An evaluation of the international experience of minimum wages in an economic downturn. Research Report for the Low Pay Commission, Royal Holloway, University of London (2011)

Dube, A., Lester, T., Reich, M.: Minimum wage effects across state borders: estimates using contiguous counties. Rev. Econ. Stat. 92(4), 945-964 (2010)

Göddeke, A., Haucap, J., Herr, A., Wey, C.: Stabilität und Wandel von Arbeitsmarktinstitutionen aus wettbewerbsökonomischer Sicht. DICE Ordnungspolitische Perspektiven (10) (2011)
Haucap, J., Pauly, U., Wey, C.: Collective wage setting when wages are generally binding. An antitrust perspective. Int. Rev. Law Econ. 21(3), 287-307 (2001)

Hethey-Maier, T., Seth, S.: Das Bertries-Historik-Panel (BHP) 19752008: Handbook Version 1.0.2. FDZ Datenreport 4, IAB Nürnberg (2010)

König, M., Möller, J.: Minimum wage effects of the worker posting law? A micro data analysis for the German construction sector. J. Labour Mark. Res. 41(2/3), 327-346 (2008)

Lee, D.: Wage inequality in the United States during the 1980s: rising dispersion or falling minimum wage? Q. J. Econ. 114(3), 9771023 (1999)

Little, R., Rubin, D.: In: Statistical Analysis with Missing Data. Wiley Series in Probability and Statistics (2002)

Manning, A.: Monopsony in Motion: Imperfect Competition in Labor Markets. Princeton University Press, Princeton (2003)

Michl, T.: Book review of myth and measurement: the new economics of the minimum wage by David Card and Alan B. Krueger. East. Econ. J. 22(2), 1996 (1996)

Neumark, D., Wascher, W.: Employment effects of minimum and subminimum wages: panel data on state minimum wage laws. Industrial and Labor Relations Review, pp. 55-81 (1992)

Neumark, D., Wascher, W.: Minimum Wages. MIT Press, Cambridge (2008)

Rattenhuber, P.: Building the minimum wage. Germany's first sectoral minimum wage and its impact on wages in the construction industry. DIW Discussion Papers 1111, Berlin (2011)

Teulings, C.: Aggregation bias in elasticities of substitution and the minimum wage paradox. Int. Econ. Rev. 41(2), 359-398 (2000)

Bodo Aretz Jahrgang 1984, hat Volkswirtschaftslehre an der Universität Bonn und an der Universität Granada in Spanien studiert. Seit Oktober 2008 ist er in Mannheim als Wissenschaftlicher Mitarbeiter am Zentrum für europäische Wirtschaftsforschung (ZEW) im Forschungsbereich „Arbeitsmärkte, Personalmanagement und Soziale Sicherung“ beschäftigt. Seine Kernforschungsthemen umfassen Arbeitsmarktpolitik, insbesondere im Niedriglohnbereich, und experimentelle Wirtschaftsforschung. Zudem hat Bodo Aretz in zahlreichen Projekten mitgewirkt, die u. a. im Auftrag des Bundesministeriums für Arbeit und Soziales, des Bundesministeriums der Finanzen und des Bundesministeriums für Wirtschaft und Technologie entstanden sind.

Bodo Aretz, born in 1984, studied Economics at the Rheinische Friedrich-Wilhelms-University in Bonn and at the University of Granada, Spain. Since October 2008, he is employed at the Centre for European Economic Research (ZEW) in Mannheim in the Research Department of Labour Markets, Human Resources and Social Policy. His main research tasks include labour market policy, the economics of wages and experimental economics. Bodo Aretz has been involved in several projects on behalf of the German Federal Ministry of Labour and Social Affairs, the Federal Ministry of Economics and Technology and the Federal Ministry of Finance.

Melanie Arntz studied geography with a focus on geographical labour markets at the University of Bonn and at the University of Minnesota. In 2007, she finished her doctoral thesis in economics at TU Darmstadt. Currently, she is deputy head of the Department of Labour Markets, Human Resources, and Social Policy at ZEW Mannheim and assistant professor for "Labour Markets" at the University of Heidelberg. Her research focus is on the dynamics of individual labour market careers and on the economic performance of regional labour markets.

Melanie Arntz studierte Geographie mit einem Schwerpunkt im Bereich regionaler Arbeitsmärkte an der Universität Bonn und der University of Minnesota. Ihre Doktorarbeit im Bereich der empirischen Wirtschaftsforschung zur räumlichen Mobilität von Arbeitskräften in Deutschland an der TU Darmstadt schloss sie 2007 ab. Derzeit ist sie 
stellvertretende Leiterin des Forschungsbereichs Arbeitsmärkte, Personalmanagement und soziale Sicherung am ZEW in Mannheim sowie Juniorprofessorin an der Universität Heidelberg für „Arbeitsmärkte“. Die Dynamik individueller Erwerbsverläufe gehört ebenso zu den Schwerpunkten ihrer Forschung wie die Entwicklung regionaler Arbeitsmärkte.

Terry Gregory studierte Volkswirtschaftslehre an der Universität Bonn und an der Karls-Universität Prag. Seine Studienschwerpunkte lagen dabei in den Bereichen Wirtschaftspolitik, Arbeitsmarktökonomik und Angewandte Mikroökonometrie. Seit März 2009 ist er am ZEW als wissenschaftlicher Mitarbeiter im Forschungsbereich Arbeitsmärkte, Personalmanagement und Soziale Sicherung tätig. Zu seinen Forschungsinteressen zählen die Arbeitskräftemigration, Mindestlöhne sowie die ökonomische Entwicklung regionaler Arbeitsmärkte. Terry Gregory hat unter anderen an Projekten für das Bundesministerium für Arbeit und Soziales mitgewirkt sowie ein Forschungsaufenthalt an der Universität Bologna absolviert.

Terry Gregory studied economics at the University of Bonn and at the Charles University in Prague focussing on economic policy, labour economics and applied microeconometrics. In March 2009 he joined the ZEW as a researcher in the Department of Labour Markets, Human Resources and Social Policy. His research focus is on labour migration, minimum wages and the economic performance of regional labour markets. Terry Gregory has been involved in projects on behalf of the German Ministry of Labour and Social Affairs and has completed a research stay at the University of Bologna.
Christian Rammer is senior research in the Department of Industrial Economics and International Management at ZEW. His main research fields are innovation economics, technology transfer and research policy. Christian Rammer is the director of ZEW's annual innovation survey (Mannheim Innovation Panel). He earned a PhD in regional science from the University of Vienna. Before joining ZEW in 2000, he worked as assistant professor at the Department of Economic Geography and Geoinformatics at the Vienna University of Economics and Business Administration and was senior researcher at the Austrian Research Centers (now Austrian Institute of Technology).

Dr. Christian Rammer ist Projektleiter im Forschungsbereich Industrieökonomik und Internationale Unternehmensführung des ZEW. Zu seinen Arbeitsschwerpunkten zählen Studien zur Innovationsforschung, zum Wissenstransfer Wirtschaft-Wissenschaft und zur Forschungsund Innovationspolitik. Christian Rammer leitet die jährliche Innovationserhebung des ZEW (Mannheimer Innovationspanel). Nach dem Studium der Regionalwissenschaften an der Universität Wien und der TU Wien war Herr Rammer von 1991 bis 1995 wissenschaftlicher Mitarbeiter am Institut für Wirtschaftsgeographie und Geoinformatik der Wirtschaftsuniversität Wien. Nach seiner Promotion wechselte er in den Forschungsbereich ,Systemforschung Technik-WirtschaftUmwelt" der Austrian Research Centers (heute: Austrian Institute of Technology). Seit April 2000 arbeitet Herr Rammer am ZEW. 